\title{
HSV-2 and Substance Abuse amongst Adolescents: Insights through Mathematical Modelling
}

\author{
A. Mhlanga, C. P. Bhunu, and S. Mushayabasa \\ Department of Mathematics, University of Zimbabwe, P.O. Box MP 167 Mount Pleasant, Harare, Zimbabwe \\ Correspondence should be addressed to C. P. Bhunu; cpbhunu@gmail.com
}

Received 5 July 2014; Accepted 5 October 2014; Published 13 November 2014

Academic Editor: Peter G. L. Leach

Copyright (C) 2014 A. Mhlanga et al. This is an open access article distributed under the Creative Commons Attribution License, which permits unrestricted use, distribution, and reproduction in any medium, provided the original work is properly cited.

\begin{abstract}
Herpes simplex virus infection is mostly spread and occurs more commonly among substance abusing adolescents as compared to the nonsubstance abusing. In this paper, a mathematical model for the spread of HSV-2 within a community with substance abusing adolescents is developed and analysed. The impacts of condom use and educational campaigns are examined. The study suggests that condom use is highly effective among adolescents, when we have more of them quitting than becoming substance abusers. Measures such as educational campaigns can be put in place to try and reduce adolescents from becoming substance abusers. Further, we applied optimal control theory to the proposed model. The controls represent condom use and educational campaigns. The objective is based on maximising the susceptible nonsubstance abusing adolescents, while minimising the susceptible substance abusing adolescents, the infectious nonsubstance abusing adolescents, and the infectious substance abusing adolescents. We used Pontrygin's maximum principle to characterise the optimal levels of the two controls. The resulting optimality system is solved numerically. Overall, the application of the optimal control theory suggests that more effort should be devoted to condom use as compared to educational campaigns.
\end{abstract}

\section{Introduction}

Herpes simplex virus type 2 (HSV-2), a double-stranded DNA virus, is a sexually transmitted disease that inflicts severe public health burden globally. The most common symptoms of HSV-2 usually appear within 1-2 weeks after sexual exposure to the virus. The first signs are a tingling sensation in the affected areas (genitalia, buttocks, and thighs) and group of small red bumps that develop into blisters. Herpes simplex virus-2 (HSV-2) is a highly prevalent STD that causes severe public health burden globally, with the highest prevalence in the Sub-Saharan Africa and some Asian countries. There are 1.5 million new herpes simplex virus2 (HSV-2) infections among 15-19 year old females in SubSaharan African every year [1]. In 2003, for instance, up to 536 million people aged 15-49 years were living with HSV2 globally and 23.6 million new infections were recorded in that year [2]. In the United States of America (USA), annual health costs for STIs has reached US \$17 Billion [3], with HSV2 chewing up $\$ 541$ million, making it the third most costly STI after HIV-1 and human papillomavirus (HPV) [4].
Adolescents are the range of people who are 10-19 years old [5], making up approximately $20 \%$ of the world's population and about $85 \%$ living in developing countries [6]. During the period of adolescence, many individuals begin to engage in risky sexual behaviours, hence making them the age group at greatest risk for nearly all STIs [7]. The reasons for this trend are many, including cognitive development, physiological susceptibility, peer pressure, logistic issues, and specific sexual behaviours [7]. Adolescents aged 15-19 years account for approximately 3 million cases, meaning one out of four sexually active teenagers reports an STI every year $[8,9]$. Adolescents are more vulnerable to STIs because of lack of education, yet, in some schools, sex education in schools begins late when adolescents have already been initiated into sexual intercourse [10]. Biologically, adolescent girls are at higher risk of contracting STIs because, in adolescent girls, the cervix and vagina undergo dramatic histological changes due to estrogen exposure [11]. Adolescent girls are also the ones at greater risk of HSV-2 infections [12, 13]. Some studies have found out that being young at age really 
increased the likelihood of acquiring HSV-2 infection [1416], implying that we have more adolescents being infected. Prevalence estimates for adolescents are available through community-based studies. One study in Zimbabwe among adolescent females aged 15-19 years reported that $12 \%$ of participants tested positive for HSV-2 [17]. A study in western Kenya found HSV-2 prevalence to be $9 \%$ for females and $4 \%$ for males aged $13-14$ years and $28 \%$ females and $17 \%$ for males aged 15-19 years [18]. It was shown that sexual activity in Namibia begins at a very young age, often as low as 10 years [10], and also some $61 \%$ percent of twelfth graders in United States of America (USA) have had sexual intercourse at least once [19]. Many adolescents experiment with substances, parents, teachers, and policy makers want to know the consequences of adolescent substance abuse. Since the abuse of substances affects their thinking capacity, most of them who are substance abusers have higher rates of HSV2 infection $[15,20]$. Those adolescents who are infected with HSV-2 type 2 (HSV-2) infection may be at greater risk for transmission and acquisition of human immunodeficiency virus (HIV) [21]. Interventions to control HSV-2 infection could prevent as many as $50 \%$ to $60 \%$ of new HIV infections [22]. Furthermore, pregnant adolescents infected with genital HSV (particularly those with a primary infection) can transmit infection to the neonate, which can lead to serious complications for the neonate, such as neurologic problems and even death $[23,24]$. Since currently there is no vaccination for HSV-2 [25], current intervention options for controlling HSV-2 are limited to patient education and promotion of condom use or treatment with oral medication [26]. Some preventive efforts should be made to target children before initiation of sexual activity, since prevention is always better than cure.

A number of mathematical models have looked into mathematical modelling of HSV-2 transmission dynamics focusing on a number of different issues (see [36-42], to mention just a few, but none has looked into the effects of substance abuse on the transmission dynamics of HSV-2 among adolescents). In contrast to other STIs such as chlamydia, gonorrhea, syphilis, and trichomoniasis, infection with HSV-2 is lifelong, and, once established, there is currently no treatment to eliminate $[43,44]$. The other problem with HSV-2 infection in adolescents is that it is somewhat difficult to determine given that many infections are asymptomatic or go unrecognised; hence it differs with most of other STIs in general. To the best of our knowledge as authors, no mathematical model has looked into the transmission dynamics of HSV-2 and substance abuse with a focus on adolescents. It is against this background that our study finds relevance and motivation, by formulating a mathematical model to investigate the impact of substance abuse among adolescents on the transmission dynamics of HSV-2. The model incorporates some key epidemiological features such as the impact of condom use and educational campaigns among the adolescents. The main objective in this study is to forecast future trends in the incidence of HSV-2 epidemic and also to quantify the association between substance abuse and HSV-2 epidemic amongst adolescents in the community.
The paper is structured as follows. The HSV-2 transmission model is formulated in the next section. Analytic results of the model system are presented in Section 3. Simulation results and projection profiles of HSV-2 are presented in Section 4. In Section 5 optimal control theory has been applied to the model formulation in Section 2. Summary and concluding remarks round up the paper.

\section{Model Formulation}

The total sexually active population at time $t$, denoted by $N$, is subdivided into mutually exclusive compartments, namely, susceptible adolescents who are nonsubstance abusers $\left(S_{n}\right)$, susceptible adolescents who are substance abusers $\left(S_{a}\right)$, adolescents infected with HSV-2 who are nonsubstance abusers $\left(I_{n}\right)$, and adolescents infected with HSV-2 who are substance abusers $\left(I_{a}\right)$. Although there are many causes of substance abuse amongst adolescents here we dwell on peer pressure as the main one. The total population is given by

$$
N=S_{n}(t)+S_{a}(t)+I_{n}(t)+I_{a}(t) \text {. }
$$

The susceptible is increased by a constant inflow into the population at rate $\Lambda$. A fraction $\pi_{0}$ of these adolescents are assumed to be nonsubstance abusers and the complementary fraction $\pi_{1}=1-\pi_{0}$ being substance abusers. This is reasonable because the underlying population is made of adolescents only into these two classes (influence from some peers is probably the main drive behind the adolescents abusing substances and hence their recruitment into the substance abusers class). It has been found that adolescents start abusing substances for four main reasons: to improve their mood, to receive social rewards, to reduce negative feelings, and to avoid social rejection [45], and these make them fall victims to peer influence easily. Individuals in different human subgroups suffer from natural death, at rate $\mu$. Assuming homogeneous mixing of the population, the susceptible individuals acquire HSV-2 infection at rate $\lambda$, with

$$
\lambda(t)=\frac{\beta\left(I_{a}(t)+(1-\eta) I_{n}(t)\right)}{N(t)} .
$$

$\eta \in(0,1)$ is a modification parameter accounting for reduced number a nonsubstance abuser would infect as compared to a substance abuser $[15,20]$, perhaps because adolescents who engage in substance abuse also engage in other risky behaviours. Substance abuse diminishes judgement, leading to the engagement in the high risk behaviours [46], and the same explanation also applies for the modification parameter $\alpha \in(0,1) . p$ is the probability of being infected from a sexual partner and $c$ is the rate at which an individual acquires sexual partners per unit time and $\beta=p c$ is the effective contact rate for HSV-2 infection (contact sufficient to result in HSV-2 infection). The role of condom use is represented by $\theta$. If $\theta=0$, then condom use is not effective; $\theta=1$ corresponds to completely effective condom use, while $0<$ $\theta<1$ implies that condom use would be effective to some degree. Substance abusers experience related diseases like liver cirrhosis, pancreatitis, and heart disease, among other 


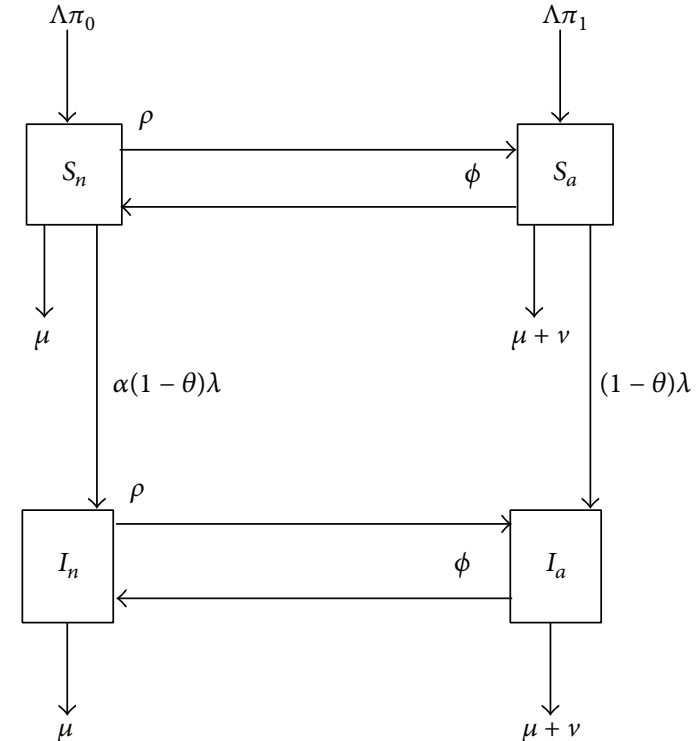

Figure 1: Structure of the model.

substance abuse induced diseases and die at a rate $v$ because of these diseases [28]. Individuals are assumed to become substance abusers mostly by peer pressure, maybe by choice, or any other reasons at rate $\rho$, and likewise individuals may cease being substance abusers due to educational campaigns (mostly), health reasons, pressure at work or school, poverty, or any other reason at rate $\phi$. The model flow diagram is depicted in Figure 1.

From the descriptions and assumptions on the dynamics of the epidemic made above, the following are the model equations:

$$
\begin{gathered}
S_{n}^{\prime}=\pi_{0} \Lambda-\alpha(1-\theta) \lambda S_{n}+\phi S_{a}-(\mu+\rho) S_{n}, \\
S_{a}^{\prime}=\pi_{1} \Lambda-(1-\theta) \lambda S_{a}+\rho S_{n}-(\mu+v+\phi) S_{a}, \\
I_{n}^{\prime}=\alpha(1-\theta) \lambda S_{n}+\phi I_{a}-(\mu+\rho) I_{n}, \\
I_{a}^{\prime}=(1-\theta) \lambda S_{a}+\rho I_{n}-(\mu+v+\phi) I_{a} .
\end{gathered}
$$

2.1. Positivity and Boundedness of Solutions. Model system (3) describes human population and therefore it is necessary to prove that all the variables $S_{n}(t), S_{a}(t), I_{n}(t)$, and $I_{a}(t)$ are nonnegative for all time. Solutions of the model system (3) with positive initial data remain positive for all time $t \geq 0$ and are bounded in $\mathscr{G}$.

Theorem 1. Let $S_{n}(t) \geq 0, S_{a}(t) \geq 0, I_{n}(t) \geq 0$, and $I_{a}(t) \geq 0$. The solutions $S_{n}, S_{a}, I_{n}$, and $I_{a}$ of model system (3) are positive for all $t \geq 0$. For model system (3), the region $\mathscr{G}$ is positively invariant and all solutions starting in $\mathscr{G}$ approach enter or stay in $\mathscr{G}$.

Proof. Under the given conditions, it is easy to prove that each solution component of model system (3) is positive; otherwise assume by contradiction that there exists a first time

$$
\begin{aligned}
t_{1}: & S_{n}\left(t_{1}\right)=0, S_{n}^{\prime}\left(t_{1}\right)<0 \text { and } S_{n}(t)>0, S_{a}(t)>0, I_{n}(t)> \\
& 0 I_{a}(t)>0 \text { for } 0<t<t_{1} \text { or there exists a, } \\
t_{2}: & S_{a}\left(t_{2}\right)=0, S_{n}^{\prime}\left(t_{2}\right)<0 \text { and } S_{n}(t)>0, S_{a}(t)>0, I_{n}(t)> \\
& 0 I_{a}(t)>0 \text { for } 0<t<t_{2} \text { or there exists a, } \\
t_{3}: & I_{n}\left(t_{3}\right)=0, I_{n}^{\prime}\left(t_{3}\right)<0 \text { and } S_{n}(t)>0, S_{a}(t)>0, I_{n}(t)> \\
& 0 I_{a}(t)>0 \text { for } 0<t<t_{3} \text { or there exists a, } \\
t_{4}: & I_{a}\left(t_{4}\right)=0, I_{a}^{\prime}\left(t_{4}\right)<0 \text { and } S_{n}(t)>0, S_{a}(t)>0, I_{n}(t)> \\
& 0 I_{a}(t)>0 \text { for } 0<t<t_{4} \text {. In the first case we have, } \\
& S_{n}^{\prime}\left(t_{1}\right)=\pi_{0} \Lambda+\phi S_{a}\left(t_{1}\right)>0,
\end{aligned}
$$

which is a contradiction and consequently $S_{n}$ remains positive. In the second case we have

$$
S_{a}^{\prime}\left(t_{2}\right)=\pi_{1} \Lambda+\rho S_{n}\left(t_{2}\right)>0,
$$

which is a contradiction; therefore $S_{a}$ is positive. In the third case we have

$$
I_{n}^{\prime}\left(t_{3}\right)=\alpha(1-\theta) \lambda\left(t_{3}\right) S_{n}\left(t_{3}\right)+\phi I_{a}\left(t_{3}\right)>0,
$$

which is a contradiction, therefore $I_{n}$ is positive. In the final case we have

$$
I_{a}^{\prime}\left(t_{4}\right)=(1-\theta) \lambda\left(t_{4}\right) S_{a}\left(t_{4}\right)+\rho I_{s}\left(t_{4}\right)>0,
$$

which is a contradiction; that is, $I_{a}$ remains positive. Thus, in all cases $S_{n}, S_{a}, I_{n}$, and $I_{a}$ remain positive.

Since $N(t) \geq S_{a}(t)$ and $N(t) \geq I_{a}(t)$, then

$$
\Lambda-(\mu+2 v) N \leq N^{\prime}(t) \leq \Lambda-\mu N
$$

implies that $N(t)$ is bounded and all solutions starting in $\mathscr{G}$ approach enter or stay in $\mathscr{G}$.

For system (3), the first octant in the state space is positively invariant and attracting; that is, solutions that start in this octant where all the variables are nonnegative stay there. Thus, system (3) will be analysed in a suitable region $\mathscr{G} \subset \mathbb{R}_{+}^{4}$

$$
\mathscr{G}=\left\{\left(S_{n}, S_{a}, I_{n}, I_{a}\right) \in \mathbb{R}_{+}^{4}: N \leq \frac{\Lambda}{\mu}\right\},
$$

which is positively invariant and attracting. It is sufficient to consider solutions in $\mathscr{G}$ where existence, uniqueness, and continuation results for system (3) hold.

\section{Model Analysis}

3.1. Disease-Free Equilibrium and Its Stability Analysis. Model system (3) has the disease-free equilibrium:

$$
\begin{aligned}
\mathscr{E}^{0}= & \frac{\Lambda\left(\pi_{0}(\mu+v)+\phi\right)}{v(\mu+\rho)+\mu(\mu+\rho+\phi)}, \\
& \left.\frac{\Lambda\left(\mu \pi_{1}+\rho\right)}{v(\mu+\rho)+\mu(\mu+\rho+\phi)}, 0,0\right) .
\end{aligned}
$$


The linear stability of $\mathscr{E}^{0}$ is governed by the basic reproductive number $R_{0}$ which is defined as the spectral radius of an irreducible or primitive nonnegative matrix [47]. Biologically, the basic reproductive number can be interpreted as the expected number of secondary infections produced by a single infectious individual during his/her infectious period when introduced in a completely susceptible population. Using the notation in [48], the nonnegative matrix $F$ and the nonsingular matrix $V$ for the new infection terms and the remaining transfer terms are, respectively, given (at DFE) by

F

$$
\begin{gathered}
{\left[\begin{array}{cc}
\frac{\alpha \beta(1-\theta)(1-\eta)\left(\pi_{0}(\mu+v)+\phi\right)}{\mu+\rho+\phi+v \pi_{0}} & \frac{\alpha \beta(1-\theta)\left(\pi_{0}(\mu+v)+\phi\right)}{\mu+\rho+\phi+v \pi_{0}} \\
\frac{\beta(1-\theta)(1-\eta)\left(\mu \pi_{1}+\rho\right)}{\mu+\rho+\phi+v \pi_{0}} & \frac{\beta(1-\theta)\left(\mu \pi_{1}+\rho\right)}{\mu+\rho+\phi+v \pi_{0}}
\end{array}\right],} \\
V=\left[\begin{array}{cc}
\mu+\rho & -\phi \\
-\rho & \mu+\phi+v
\end{array}\right] .
\end{gathered}
$$

We now consider different possibilities in detail.

Case $a$ (there are no substance abusers in the community). We set $v=\phi=\rho=\pi_{1}=0$ and $\pi_{0}=1$. Then the spectral radius is given by

$$
R_{0}=R_{n}=\frac{\alpha \beta(1-\theta)(1-\eta)}{\mu} .
$$

This reproductive rate sometimes referred to as the back of the napkin [49] is simply the ratio of the per capita rate of infection and the average lifetime of an individual in class $I_{n}$. It is defined as the number of secondary HSV-2 cases produced by a single infected individual during his/her entire infectious period in a totally naive (susceptible) population in the absence of substance abusers.

Case $b$ (the entire population is made up of substance abusers). We set $\phi=\rho=\pi_{0}=0$ and $\pi_{1}=1$. Then the spectral radius is given by

$$
R_{a}=\frac{\beta(1-\theta)}{\mu+v}
$$

biologically, $R_{a}$ measures the average number of new infections generated by a single HSV-2 infective who is also a substance abuser during his/her entire infectious period when he/she is introduced to a susceptible population of substance abusers.

Case $c$ (there is no condom use). We set $\theta=0$. Then the spectral radius is given by

$$
R_{c}=\frac{\beta\left(k_{1}+k_{2}+k_{3}\right)}{\left(\mu+\rho+\phi+v \pi_{0}\right)((\mu+v)(\mu+\rho)+\mu \phi)},
$$

where

$$
\begin{aligned}
& k_{1}=v \alpha\left((1-\eta)\left(2 \mu \pi_{0}+\phi\right)+\pi_{0}(\rho+(1-\eta) \phi)\right) \\
& k_{2}=\left(\mu \pi_{1}+\rho\right)(\mu+\rho+(1-\eta) \phi)+v^{2} \alpha(1-\eta) \pi_{0}, \\
& k_{3}=\alpha\left(\mu \pi_{0}+\phi\right)(\rho+(1-\rho)(\mu+\phi)+\rho)
\end{aligned}
$$

biologically, $R_{c}$ measures the average number of new secondary HSV-2 infections generated by a single HSV-2 infective during his/her infectious period whilst in a community of nonsubstance abusers and substance abusers, in the absence of condom use.

Case $d$ (the general case). Existence of nonsubstance abusers and substance abusers. Then the spectral radius is given by

$$
R_{n a}=\frac{\beta(1-\theta)\left(k_{1}+k_{2}+k_{3}\right)}{\left(\mu+\rho+\phi+v \pi_{0}\right)((\mu+v)(\mu+\rho)+\mu \phi)},
$$

where

$$
\begin{aligned}
& k_{1}=v \alpha\left((1-\eta)\left(2 \mu \pi_{0}+\phi\right)+\pi_{0}(\rho+(1-\eta) \phi)\right), \\
& k_{2}=\left(\mu \pi_{1}+\rho\right)(\mu+\rho+(1-\eta) \phi)+v^{2} \alpha(1-\eta) \pi_{0}, \\
& k_{3}=\alpha\left(\mu \pi_{0}+\phi\right)(\rho+(1-\rho)(\mu+\phi)+\rho) ;
\end{aligned}
$$

biologically, $R_{n a}$ measures the average number of new HSV2 infections generated by a single HSV-2 infective during his/her infectious period within a community, in the presence of condom use, nonsubstance abusers, and substance abusers. Theorem 2 follows from van den Driessche and Watmough [48].

Theorem 2. The disease-free equilibrium of model system (3) is locally asymptotically stable if $R_{n a} \leq 1$, and unstable otherwise.

Using a proof based on the comparison theorem, we can even show the global stability of the disease-free equilibrium in the case that the disease-free equilibrium is less than unity.

\subsubsection{Global Stability of the Disease-Free Equilibrium}

Theorem 3. The disease-free equilibrium $\mathscr{E}^{0}$ of system (3) is globally asymptotically stable (GAS) if $R_{n a} \leq 1$ and unstable if $R_{n a}>1$.

Proof. The proof is based on using a comparison theorem [50]. Note that the equations of the infected components in system (3) can be written as

$$
\left[\begin{array}{l}
\frac{d I_{n}}{d t} \\
\frac{d I_{a}}{d t}
\end{array}\right]=[F-V]\left[\begin{array}{l}
I_{n} \\
I_{a}
\end{array}\right]-H\left[\begin{array}{cc}
1-\eta & 1 \\
0 & 0
\end{array}\right]\left[\begin{array}{l}
I_{n} \\
I_{a}
\end{array}\right]
$$

where $F$ and $V$ are as defined earlier in (11) and (12), respectively, and also where

$$
\begin{aligned}
H=\left[\alpha \beta(1-\theta)\left(\frac{\pi_{0}(\mu+v)+\phi}{\mu+\phi+\rho+v \pi_{0}}-\frac{S_{n}}{N}\right)\right. \\
\left.\quad+\beta(1-\theta)\left(\frac{\mu \pi_{1}+\rho}{\mu+\phi+\rho+v \pi_{0}}-\frac{S_{a}}{N}\right)\right] .
\end{aligned}
$$


Since $H \geq 0$ (full proof for $H \geq 0$ : see Appendix A) (for all time $t \geq 0)$ in $\mathscr{G}$, it follows that

$$
\left[\begin{array}{l}
\frac{d I_{n}}{d t} \\
\frac{d I_{a}}{d t}
\end{array}\right] \leq[F-V]\left[\begin{array}{l}
I_{n} \\
I_{a}
\end{array}\right]
$$

Using the fact that the eigenvalues of the matrix $F-V$ all have negative real parts, it follows that the linearized differential inequality system (21) is stable whenever $R_{n a} \leq 1$. Consequently, $\left(I_{n}, I_{a}\right) \rightarrow(0,0)$ as $t \rightarrow \infty$. Thus, by a comparison theorem [50] $\left(I_{n}, I_{a}\right) \rightarrow(0,0)$ as $t \rightarrow \infty$, and evaluating system (3) at $I_{n}=I_{a}=0$ gives $S_{n} \rightarrow S_{n}^{0}$ and $S_{a} \rightarrow S_{a}^{0}$ for $R_{n a} \leq 1$. Hence, the disease-free equilibrium (DFE) is globally asymptotically stable for $R_{n a} \leq 1$.

3.2. Endemic Equilibrium and Stability Analysis. Model system (3) has three possible endemic equilibria: the (HSV-2 only) substance abuse-free endemic equilibria with a population of nonsubstance abusers only, the endemic equilibrium when the whole population is made up of substance abusers only and lastly the equilibrium where nonsubstance abusers and substance abusers co-exist, herein referred to as the interior equilibrium point.

3.2.1. Nonsubstance Abusers (Only) Endemic Equilibrium. We set $\pi_{1}=\rho=\phi=0$ and $\pi_{0}=1$, so that there are no substance abusers in the community. Thus, model system (3) reduces to

$$
\begin{gathered}
S_{n}^{\prime}=\Lambda-\frac{\alpha(1-\theta)(1-\eta) \beta S_{n}^{*} I_{n}^{*}}{N_{n}^{*}}-\mu S_{n}^{*}, \\
I_{n}^{\prime}=\frac{\alpha(1-\theta)(1-\eta) \beta S_{n}^{*} I_{n}^{*}}{N_{n}^{*}}-\mu I_{n}^{*} .
\end{gathered}
$$

For system (22), it can be shown that the region

$$
\mathscr{G}_{n}=\left\{\left(S_{n}, I_{n}\right) \in \mathbb{R}_{+}^{2}: N_{n} \leq \frac{\Lambda}{\mu}\right\}
$$

is invariant and attracting. Thus, the dynamics of nonsubstance abusing (only) adolescents would be considered in $\mathscr{G}_{n}$.

Summing the two equation in system (22) above, we get $N_{n}=\Lambda / \mu$. Furthermore, from the second equation of system (22), $I_{n}=0$ or

$$
\begin{aligned}
0 & =\frac{\alpha(1-\theta)(1-\eta) \beta S_{n}^{*} I_{n}^{*}}{N_{n}^{*}}-\mu I_{n}^{*} \\
& \Longrightarrow \alpha(1-\theta)(1-\eta) \beta S_{n}^{*}=\mu N_{n}^{*} \\
& \Longrightarrow S_{n}^{*}=\frac{\Lambda}{\mu R_{n}}
\end{aligned}
$$

with $R_{n}$ as defined in (13).

Since $N_{n}^{*}=S_{n}^{*}+I_{n}^{*}$, hence $I_{n}^{*}=N_{n}^{*}-S_{n}^{*}$, and thus

$$
\begin{aligned}
I_{n}^{*} & =\frac{\Lambda}{\mu}-\frac{\Lambda}{\mu R_{n}} \\
& =\left(R_{n}-1\right) S_{n}^{*} .
\end{aligned}
$$

Thus, system (22) has an endemic equilibrium $\mathscr{E}_{n}^{*}=\left(S_{n}^{*}, I_{n}^{*}\right)$ which makes biological sense only when $R_{n}>1$. This leads to Theorem 4 below.

Theorem 4. The substance abuse-free endemic equilibrium $\mathscr{E}_{n}^{*}$ exists whenever $R_{n}>1$.

The local stability of $\mathscr{E}_{n}^{*}$ is given by the Jacobian evaluated at the point:

$$
\begin{aligned}
& J\left(\mathscr{E}_{n}^{*}\right) \\
& =\left[\begin{array}{cc}
-\left(\frac{\alpha(1-\theta)(1-\eta) \beta I_{n}^{*}}{N_{n}^{*}}+\mu\right) & \frac{\alpha(1-\theta)(1-\eta) \beta S_{n}^{*}}{N_{n}^{*}} \\
\frac{\alpha(1-\theta)(1-\eta) \beta I_{n}^{*}}{N_{n}^{*}} & \frac{\alpha(1-\theta)(1-\eta) \beta S_{n}^{*}}{N_{n}^{*}}-\mu
\end{array}\right] .
\end{aligned}
$$

From (22) we note that $\alpha(1-\theta)(1-\eta) \beta S_{n}^{*} / N_{n}^{*}=\mu$, which is obtained from the second equation of system (22) with the right-hand side set equaling zero. Thus, $J\left(\mathscr{E}_{n}^{*}\right)$ can be written as

$$
J\left(\mathscr{E}_{n}^{*}\right)=\left[\begin{array}{cc}
-\left(\frac{\alpha(1-\theta)(1-\eta) \beta I_{n}^{*}}{N_{n}^{*}}+\mu\right) & -\mu \\
\frac{\alpha(1-\theta)(1-\eta) \beta I_{n}^{*}}{N_{n}^{*}} & 0
\end{array}\right] .
$$

Hence the characteristic polynomial of the linearised system is given by

$$
\begin{gathered}
\Upsilon^{2}+\left(\frac{\alpha(1-\theta)(1-\eta) \beta I_{n}^{*}}{N_{n}^{*}}+\mu\right) \Upsilon \\
+\frac{\alpha(1-\theta)(1-\eta) \mu \beta I_{n}^{*}}{N_{n}^{*}}=0 \\
\Longrightarrow \Upsilon_{1}=-\mu, \\
\Upsilon_{2}=-\alpha(1-\alpha)(1-\eta)\left(R_{N}-1\right) \frac{\beta I_{n}^{*}}{N_{n}^{*}}
\end{gathered}
$$

Thus, $\mathscr{E}_{n}^{*}$ is locally asymptotically stable for $R_{n}>1$. We summarise the result in Theorem 5 .

Theorem 5. The endemic equilibrium $\left(\mathscr{E}_{n}^{*}\right)$ is locally asymptotically stable whenever $R_{n}>1$.

We now study the global stability of system (22) using the Poincaré-Bendixon Theorem [51]. We claim the following result.

Theorem 6. The endemic equilibrium of the HSV-2 only model system (22) is globally asymptotically stable in $\mathscr{G}_{n}$ whenever $R_{n}>1$.

Proof. From model system (22), we note that $N_{n}=\Lambda / \mu$. Using the fact that $S_{n}=N_{n}-I_{n}$, it follows that $S_{n}=(\Lambda / \mu)-I_{n}$, and, substituting this result into (22), we obtain

$$
I_{n}^{\prime}=\frac{\alpha(1-\theta)(1-\eta) \beta I_{n}}{\Lambda / \mu}\left(\frac{\Lambda}{\mu}-I_{n}\right)-\mu I_{n} .
$$


Using Dulac's multiplier $1 / I_{n}$, it follows that

$$
\begin{gathered}
\frac{\partial}{\partial I_{n}}\left[\frac{\alpha(1-\theta)(1-\eta) \beta}{\Lambda / \mu}\left(\frac{\Lambda}{\mu}-I_{n}\right)-\mu\right] \\
=-\frac{\alpha(1-\theta)(1-\eta) \mu \beta}{\Lambda}<0 \\
=-\frac{\alpha(1-\theta)(1-\eta) \beta}{N}<0 .
\end{gathered}
$$

Thus, by Dulac's criterion, there are no periodic orbits in $\mathscr{G}_{n}$. Since $\mathscr{G}_{n}$ is positively invariant and the endemic equilibrium exists whenever $R_{n}>1$, then it follows from the PoincaréBendixson Theorem [51] that all solutions of the limiting system originating in $\mathscr{G}$ remain in $\mathscr{G}_{n}$, for all $t \geq 0$. Further, the absence of periodic orbits in $\mathscr{G}_{n}$ implies that the endemic equilibrium of the HSV-2 only model is globally asymptotically stable.

3.2.2. Substance Abusers (Only) Endemic Equilibrium. This occurs when the whole community consists of substance abusers only. Using the similar analysis as in Section 3.2.1, it can be easily shown that the endemic equilibrium is

$$
\mathscr{E}_{a}^{*}=\left\{S_{a}^{*}=\frac{\Lambda}{\mu R_{a}}, I_{a}^{*}=\left(R_{a}-1\right) S_{a}^{*}\right\}
$$

with $R_{a}$ as defined in (14), and it follows that the endemic equilibrium $\mathscr{E}_{a}^{*}$ makes biological sense whenever $R_{a}>1$. Furthermore, using the analysis done in Section 3.2.1, the stability of $\mathscr{E}_{a}^{*}$ can be established. We now discuss the coexistence of nonsubstance abusers and substance abusers in the community.

3.2.3. Interior Endemic Equilibrium. This endemic equilibrium refers to the case where both the nonsubstance abusers and the substance abusers coexist. This state is denoted by $\mathscr{E}_{n a}^{*}$ with

$$
\mathscr{E}_{n a}^{*}=\left\{\begin{array}{l}
S_{n}^{*}=\frac{\pi_{0} \Lambda+\phi S_{a}^{*}}{\alpha(1-\theta) \lambda_{n a}^{*}+\mu+\rho}, \\
S_{a}^{*}=\frac{\pi_{1} \Lambda+\rho S_{n}^{*}}{(1-\theta) \lambda_{n a}^{*}+\mu+v+\phi}, \\
I_{n}^{*}=\frac{\alpha(1-\theta) \lambda_{n a}^{*} S_{n}^{*}+\phi I_{a}^{*}}{\mu+\rho}, \\
I_{a}^{*}=\frac{(1-\theta) \lambda_{n a}^{*} S_{a}^{*}+\rho I_{n}^{*}}{\mu+v+\phi}, \\
\text { with } \lambda_{n a}^{*}=\frac{\beta\left(I_{a}^{*}+(1-\eta) I_{n}^{*}\right)}{N_{n a}^{*}} .
\end{array}\right.
$$

We now study the global stability of the interior equilibrium of system (3) using the Poincaré-Bendinxon Theorem (see Appendix B). The permanence of the disease destabilises the disease-free equilibrium $\mathscr{C}^{0}$ since $R_{n a}>1$, and, thus, the equilibrium $\mathscr{E}_{n a}^{*}$ exists.

Lemma 7. Model system (3) is uniformlypersistent on $\mathscr{G}$.
Proof. Uniform persistence of system (3) implies that there exists a constant $\zeta>0$ such that any solution of model (3) starts in $\dot{\mathscr{G}}$, and the interior of $\mathscr{G}$ satisfies

$$
\begin{array}{ll}
\zeta \leq \lim \inf _{t \rightarrow \infty} S_{n}, & \zeta \leq \lim \inf _{t \rightarrow \infty} S_{a}, \\
\zeta \leq \lim \inf _{t \rightarrow \infty} I_{n}, & \zeta \leq \lim \inf _{t \rightarrow \infty} I_{a} .
\end{array}
$$

Define the following Korobeinikov and Maini [52] type of Lyapunov functional:

$$
\begin{aligned}
V\left(S_{n}, S_{a}, I_{n}, I_{a}\right)= & \left(S_{n}-S_{n}^{*} \ln S_{n}\right)+\left(S_{a}-S_{a}^{*} \ln S_{a}\right) \\
& +\left(I_{n}-I_{n}^{*} \ln I_{n}\right)+\left(I_{a}-I_{a}^{*} \ln I_{a}\right)
\end{aligned}
$$

which is continuous for all $\left(S_{n}, S_{a}, I_{n}, I_{a}\right)>0$ and satisfies

$$
\begin{array}{ll}
\frac{\partial V}{\partial S_{n}}=\left(1-\frac{S_{n}^{*}}{S_{n}}\right), & \frac{\partial V}{\partial S_{a}}=\left(1-\frac{S_{a}^{*}}{S_{a}}\right), \\
\frac{\partial V}{\partial I_{n}}=\left(1-\frac{I_{n}^{*}}{I_{n}}\right), & \frac{\partial V}{\partial I_{a}}=\left(1-\frac{I_{a}^{*}}{I_{a}}\right)
\end{array}
$$

(see Korobeinikov and Wake [53] for more details). Consequently, the interior equilibrium $\mathscr{E}_{n a}^{*}$ is the only extremum and the global minimum of the function $V \in \mathbb{R}_{+}^{4}$. Also, $V\left(S_{n}, S_{a}, I_{n}, I_{a}\right)>0$ and $V^{\prime}\left(S_{n}, S_{a}, I_{n}, I_{a}\right)=0$ only at $\mathscr{E}_{n a}^{*}$. Then, substituting our interior endemic equilibrium identities into the time derivative of $V$ along the solution path of the model system (3), we have

$$
\begin{aligned}
V^{\prime}\left(S_{n}, S_{n}, I_{a}, I_{a}\right)= & \left(S_{n}-S_{n}^{*}\right) \frac{S_{n}^{\prime}}{S_{n}}+\left(S_{a}-S_{a}^{*}\right) \frac{S_{a}^{\prime}}{S_{a}} \\
& +\left(I_{n}-I_{n}^{*}\right) \frac{I_{n}^{\prime}}{I_{n}}+\left(I_{a}-I_{a}^{*}\right) \frac{I_{a}^{\prime}}{I_{a}} \\
\leq & -\mu \frac{\left(S_{n}-S_{n}^{*}\right)^{2}}{S_{n}}+g\left(S_{n}, S_{a}, I_{n}, I_{a}\right) .
\end{aligned}
$$

The function, $g$, can be shown to be nonpositive using Barbalat Lemma [54] or the approach in McCluskey [55]. Hence, $V^{\prime}\left(S_{n}, S_{a}, I_{n}, I_{a}\right) \leq 0$ with equality only at $\mathscr{E}_{n a}^{*}$. The only invariant set in $\mathscr{G}_{\text {is }}$ the set consisting of the equilibrium $\mathscr{E}_{n a}^{*}$. Thus, all solutions of model system (3) which intersect with $\stackrel{\mathscr{G}}{ }$ limit to an invariant set, the singleton $\left\{\mathscr{E}_{n a}^{*}\right\}$. Therefore, from the Lyapunov-Lasalle invariance principle, model system (3) is uniformly persistent.

From the epidemiological point of view, this result means that the disease persists at endemic state, which is not good news from the public health viewpoint.

Theorem 8. If $R_{n a}>1$, then the interior equilibrium $\mathscr{E}_{n a}^{*}$ is globally asymptotically stablein the interior of $\mathscr{G}$. 
TABLE 1: Model parameters and their interpretations.

\begin{tabular}{|c|c|c|c|}
\hline Definition & Symbol & Baseline values (range) & Source \\
\hline Recruitment rate & $\Lambda$ & $10000 \mathrm{yr}^{-1}$ & Assumed \\
\hline Proportion of recruited individuals & $\pi_{0}, \pi_{1}$ & $0.7,0.3$ & Assumed \\
\hline Natural death rate & $\mu$ & $0.02(0.015-0.02) \mathrm{yr}^{-1}$ & {$[27]$} \\
\hline Contact rate & $c$ & 3 & {$[28]$} \\
\hline HSV-2 transmission probability & $p$ & $0.01(0.001-0.03) \mathrm{yr}^{-1}$ & {$[29-32]$} \\
\hline Substance abuse induced death rate & $v$ & $0.035 \mathrm{yr}^{-1}$ & [33] \\
\hline Rate of change in substance abuse habit & $\rho, \phi$ & Variable & Assumed \\
\hline Condom use efficacy & $\theta$ & 0.5 & {$[34,35]$} \\
\hline Modification parameter & $\eta$ & $0.6(0-1)$ & Assumed \\
\hline Modification parameter & $\alpha$ & $0.4(0-1)$ & Assumed \\
\hline
\end{tabular}

Proof. Denote the right-hand side of model system (3) by $\Phi$ and $\Psi$ for $I_{n}$ and $I_{a}$, respectively, and choose a Dulac function as $D\left(I_{n}, I_{a}\right)=1 / I_{n} I_{a}$. Then we have

$$
\begin{aligned}
\frac{\partial(D \Phi)}{\partial I_{n}} & +\frac{\partial(D \Psi)}{\partial I_{a}} \\
= & -\frac{\phi}{I_{n}^{2}}-\frac{\rho}{I_{a}^{2}}-(1-\theta) \beta\left(\frac{\alpha S_{n}}{I_{n}^{2}}+\frac{(1-\eta) S_{a}}{I_{a}^{2}}\right)<0 .
\end{aligned}
$$

Thus, system (3) does not have a limit cycle in the interior of $\mathscr{G}$. Furthermore, the absence of periodic orbits in $\mathscr{G}$ implies that $\mathscr{E}_{n a}^{*}$ is globally asymptotically stable whenever $R_{n a}>1$.

\section{Numerical Results}

In order to illustrate the results of the foregoing analysis, we have simulated model (3) using the parameters in Table 1. Unfortunately, the scarcity of the data on HSV-2 and substance abuse in correlation with a focus on adolescents limits our ability to calibrate; nevertheless, we assume some of the parameters in the realistic range for illustrative purpose. These parsimonious assumptions reflect the lack of information currently available on HSV-2 and substance abuse with a focus on adolescents. Reliable data on the risk of transmission of HSV-2 among adolescents would enhance our understanding and aid in the possible intervention strategies to be implemented.

Figure 2 shows the effects of varying the effective contact rate on the reproduction number. Results on Figure 2 suggest that the increase or the existence of substance abusers may increase the reproduction number. Further analysis of Figure 2 reveals that the combined use of condoms and educational campaigns as HSV-2 intervention strategies among substance abusing adolescents in a community have an impact on reducing the magnitude of HSV-2 cases, as shown by the lowest graph for $R_{n a}$.

Figure 3(a) depicts that the reproduction number can be reduced by low levels of adolescents becoming substance abusers and high levels of adolescents quitting the substance abusive habits. Numerical results in Figure 3(b) suggest that more adolescents should desist from substance abuse, since

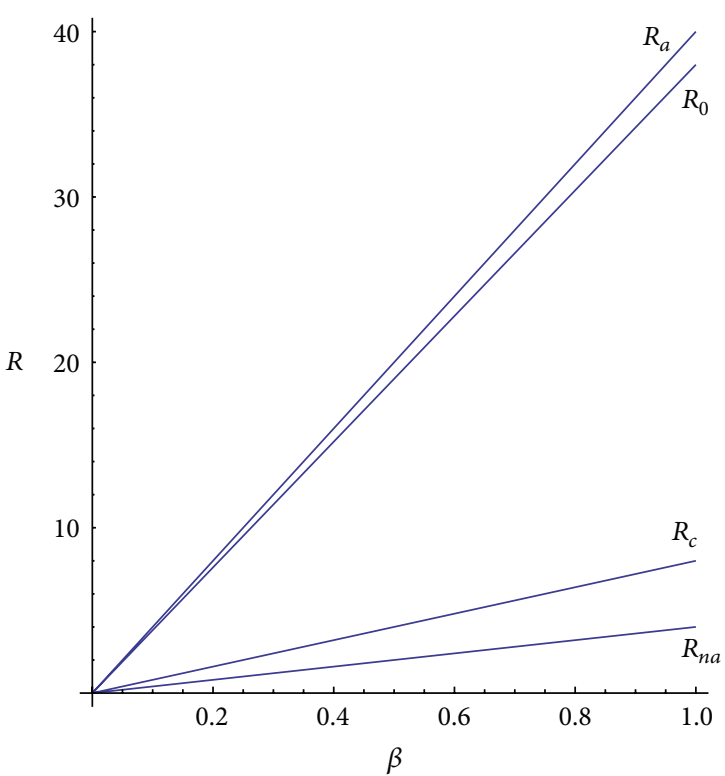

FIGURE 2: Effects of varying the effective contact rate $(\beta)$ on the reproduction numbers.

the trend $\Sigma$ shows a decrease in the reproduction number $R_{n a}$ with increasing $\phi$. Trend line $\Omega$ shows the effects of increasing $\rho$ which results in an increase of the reproductive number $R_{n a}$ thereby increasing the prevalence of HSV-2 in community. The point $\psi$ depicts the balance in transfer rates from being a substance abuser to a nonsubstance abuser or from a nonsubstance abuser to a substance abuser $(\rho=\phi)$, and this suggests that changes in the substance abusive habit will not have an impact on the reproduction number. Point $\psi$ also suggests that, besides substance abuse among adolescents, other factors are influencing the spread of HSV-2 amongst them in the community. Measures such as educational campaigns and counselling may be introduced so as to reduce $\rho$ while increasing $\phi$.

In many epidemiological models, the magnitude of the reproductive number is associated with the level of infection. The same is true in model system (3). Sensitivity analysis assesses the amount and type of change inherent in the model as captured by the terms that define the reproductive number 


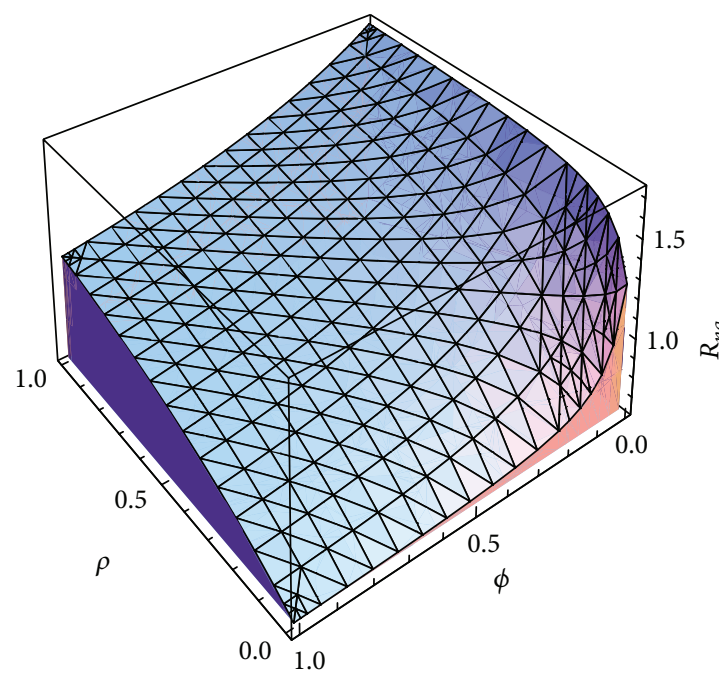

(a)

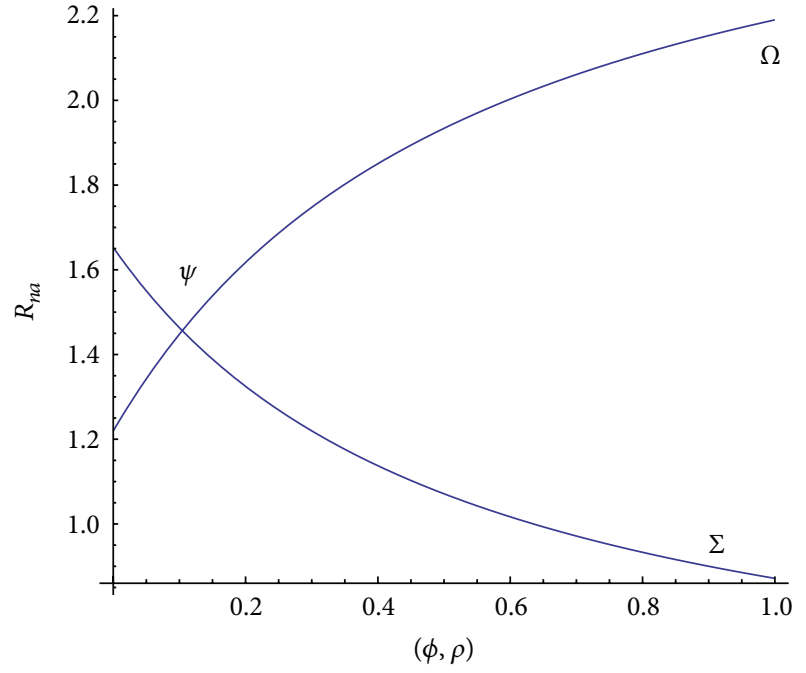

(b)

FIGURE 3: (a) The relationship between the reproduction number $\left(R_{n a}\right)$, rate of becoming a substance abuser $(\rho)$, and rate of quitting substance abuse $(\phi)$. (b) Numerical results of model system (3) showing the effect in the change of substance abusing habits. Parameter values used are in Table 1 with $\phi$ and $\rho$, varying from 0.0 to 1.0 .

$\left(R_{n a}\right)$. If $R_{n a}$ is very sensitive to a particular parameter, then a perturbation of the conditions that connect the dynamics to such a parameter may prove useful in identifying policies or intervention strategies that reduce epidemic prevalence.

Partial rank correlation coefficients (PRCCs) were calculated to estimate the degree of correlation between values of $R_{n a}$ and the ten model parameters across 1000 random draws from the empirical distribution of $R_{n a}$ and its associated parameters.

Figure 4 illustrates the PRCCs using $R_{n a}$ as the output variable. PRCCs show that the rate of becoming substance abusers by the adolescents has the most impact in the spread of HSV-2 among adolescents. Effective contact rate is found to support the spread of HSV-2 as noted by its positive PRCC. However, condom use efficacy is shown to have a greater impact on reducing $R_{n a}$.

Since the rate of becoming a substance abuser $(\rho)$, condom use efficacy $(\theta)$, and the effective contact rate $(\beta)$ have significant effects on $R_{n a}$, we examine their dependence on $R_{n a}$ in more detail. We used Latin hypercube sampling and Monte Carlo simulations to run 1000 simulations, where all parameters were simultaneously drawn from across their ranges.

Figure 5 illustrates the effect that varying three sample parameters will have on $R_{n a}$. In Figure 5(a), if the rate of becoming a substance abuser is sufficiently high, then $R_{n a}>1$ and the disease will persist. However, if the rate of becoming a substance abuser is low, then $R_{n a}>1$ and the disease can be controlled. Figure 5(b) illustrates the effect of condom use efficacy on controlling HSV-2. The result demonstrates that an increase in $\theta$ results in a decrease in the reproduction number. Figure 5(b) demonstrates that if condom use efficacy is more than $70 \%$ of the time, then the reproduction number is less than unity, and then the disease will be controlled.

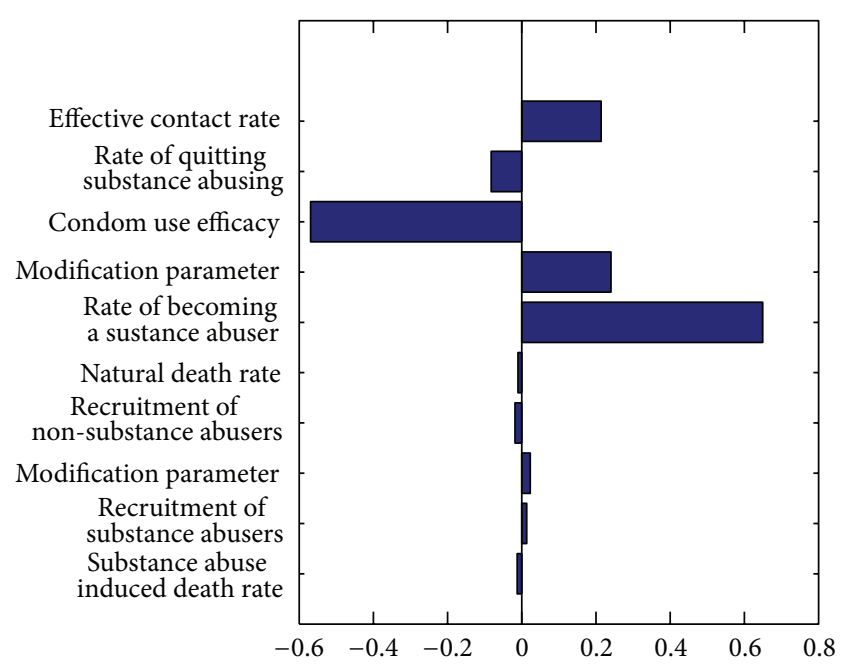

FIgURE 4: Partial rank correlation coefficients showing the effect of parameter variations on $R_{n a}$ using ranges in Table 1 . Parameters with positive PRCCs will increase $R_{n a}$ when they are increased, whereas parameters with negative PRCCs will decrease $R_{n a}$ when they are increased.

Numerical results on Figure 8 are in agreement with the earlier findings, which have shown that condom use has a significant effect on HSV-2 cases among adolescents. However, Figure 8(b) shows that, for more adolescents leaving their substance abuse habit than becoming substance abusers, condom use is more effective. Figure 8(b) also shows that more adolescents should desist from substance abuse to reduce the HSV-2 cases. It is also worth noting that, as the condom use efficacy is increasing, the cumulative HSV-2 cases also are reduced.

In general, simulations in Figure 7 suggest that an increase in recruitment of substance abusing adolescents into 


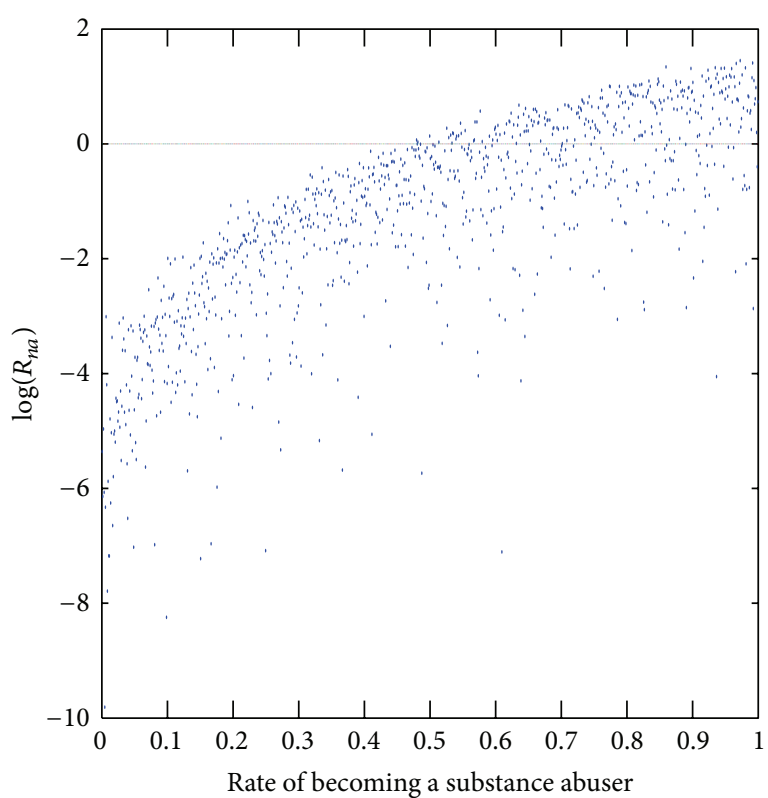

(a)

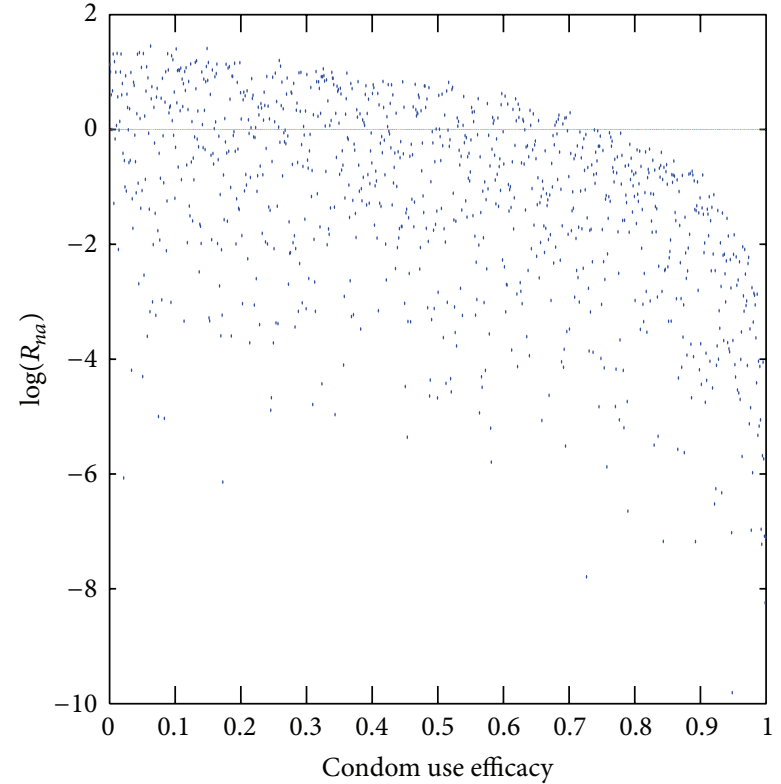

(b)

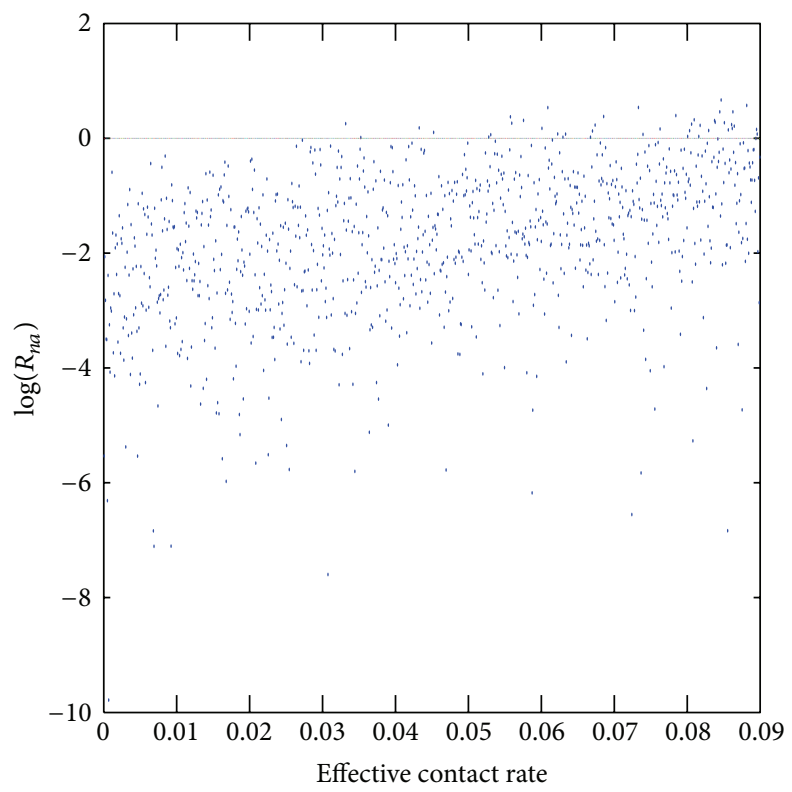

(c)

Figure 5: Monte Carlo simulations of 1000 sample values for three illustrative parameters $(\rho, \theta$, and $\beta)$ chosen via Latin hypercube sampling.

the community will increase the prevalence of HSV-2 cases. It is worth noting that the prevalence is much higher when we have more adolescents becoming substance abusers than quitting the substance abusing habit, that is, for $\rho>\phi$.

\section{Optimal Condom Use and Educational Campaigns}

In this section our goal is to solve the following problem: given initial population sizes of all the four subgroups, $S_{n}$, $S_{a}, I_{n}$, and $I_{a}$, find the best strategy in terms of combined efforts of condom use and educational campaigns that would minimise the cumulative HSV-2 cases while at the same time minimising the cost of condom use and educational campaigns. Naturally, there are various ways of expressing such a goal mathematically. In this section, for a fixed $T$, we consider the following objective functional:

$$
\begin{aligned}
& J\left(u_{1}, u_{2}\right) \\
& \quad=\int_{0}^{t_{f}}\left[S_{n}-B_{1} S_{a}-B_{2} I_{n}-B_{3} I_{a}-\left(A_{1} u_{1}^{2}+A_{2} u_{2}^{2}\right)\right] d t
\end{aligned}
$$




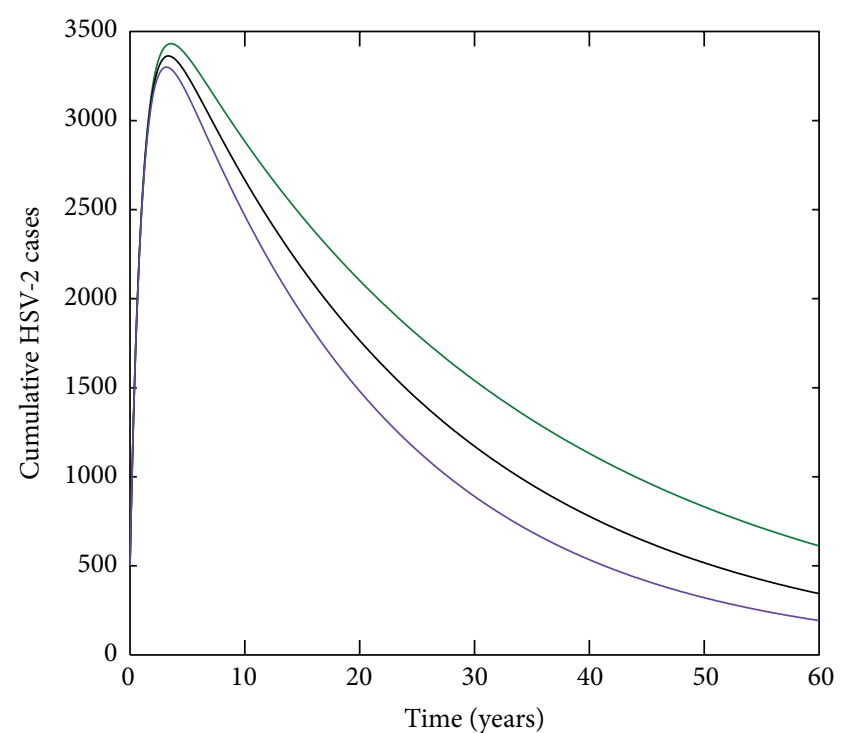

$\begin{aligned}-\theta & =0.2 \\ -\theta & =0.5 \\ -\theta & =0.8\end{aligned}$

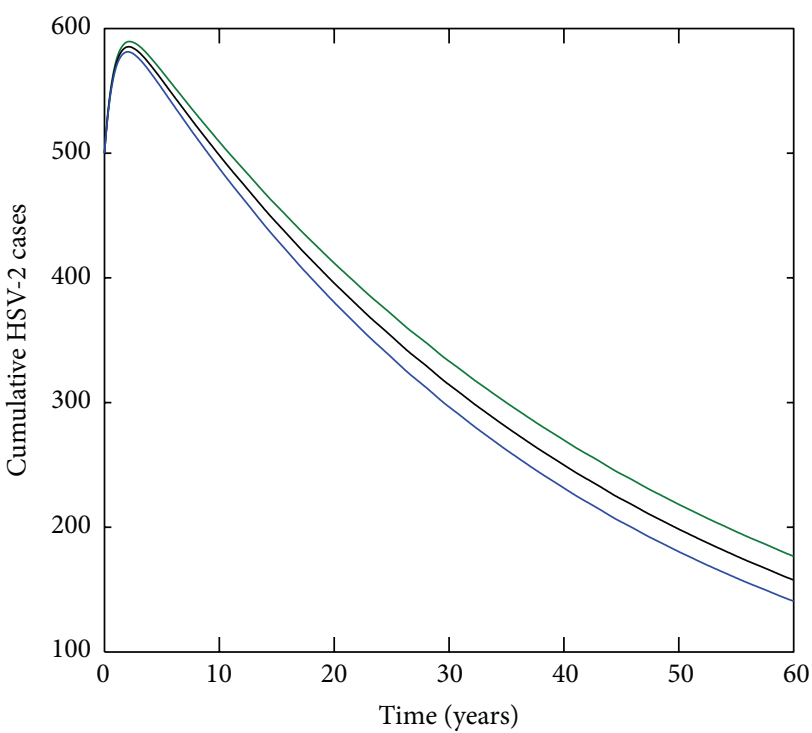

$-\theta=0.2$

$-\theta=0.5$

$-\theta=0.8$

(a)

(b)

Figure 6: Simulations of model system (3) showing the effects of an increase on condom use efficacy on the HSV-2 cases $\left(I_{a}+I_{n}\right)$ over a period of 60 years. The rest of the parameters are fixed on their baseline values, with Figure 8(a) having $\rho>\phi$ and Figure 8 (b) having $\phi>\rho$.

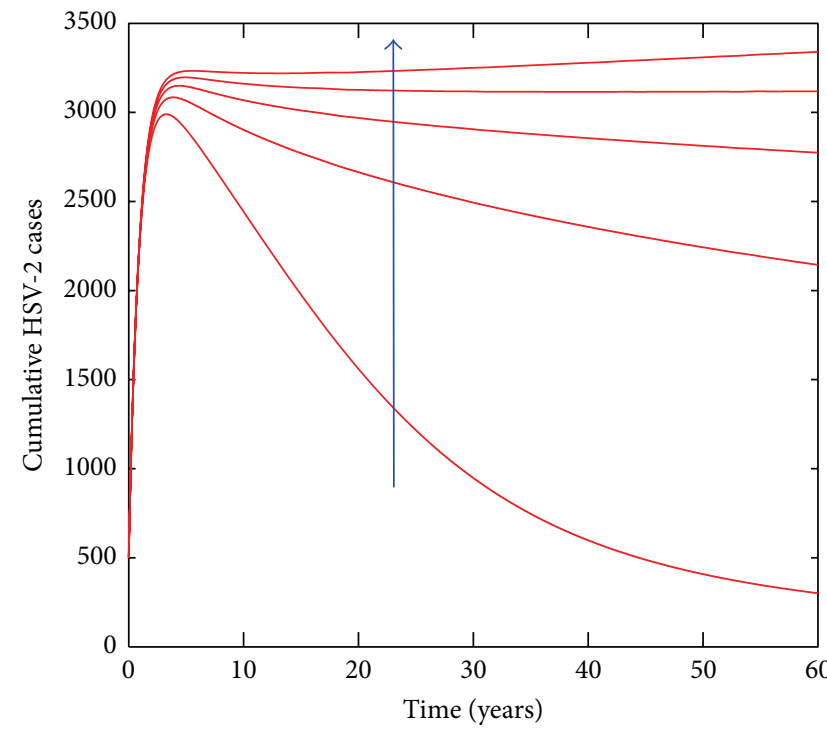

(a)

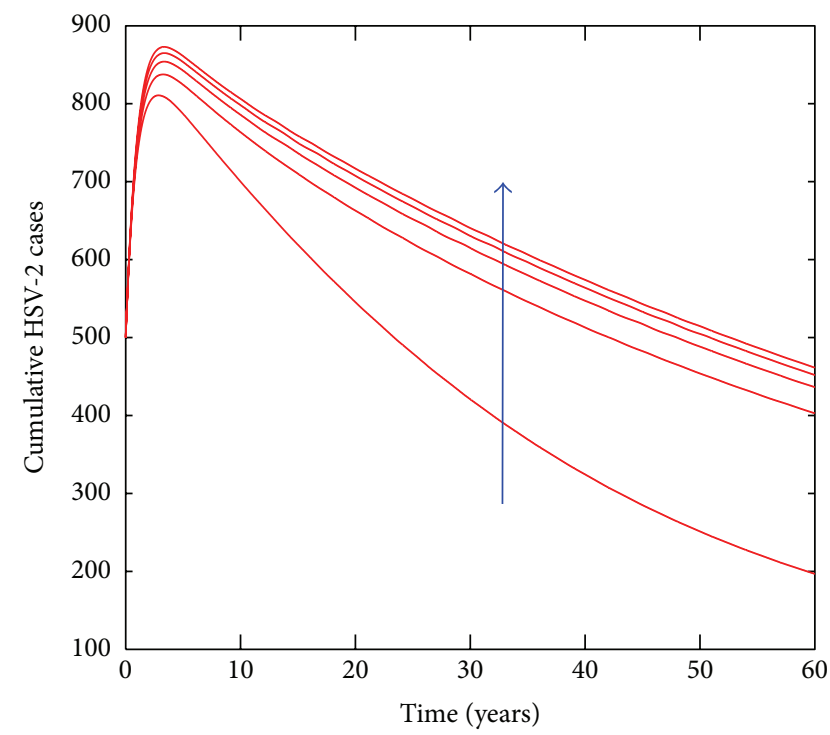

(b)

FIGURE 7: Simulations of model system (3) showing the effects of increasing the proportion of recruited substance abusers $\left(\pi_{1}\right)$, varying from 0.0 to 1.0 with a step size of 0.25 , for the 2 cases where (a) $\rho>\phi$ and (b) $\phi>\rho$.

subject to

$$
\begin{aligned}
S_{n}^{\prime}=\pi_{0} \Lambda-\alpha & \left(1-u_{1}\right) \lambda S_{n}+\phi S_{a}-\left(1-u_{2}\right) \rho S_{n}-\mu S_{n}, \\
S_{a}^{\prime}= & \left(1-u_{2}\right) \pi_{1} \Lambda-\left(1-u_{1}\right) \lambda S_{a} \\
& +\left(1-u_{2}\right) \rho S_{n}-(\mu+v+\phi) S_{a},
\end{aligned}
$$

$$
\begin{gathered}
I_{n}^{\prime}=\alpha\left(1-u_{1}\right) \lambda S_{n}+\phi I_{a}-\left(1-u_{2}\right) \rho I_{n}-\mu I_{n}, \\
I_{a}^{\prime}=\left(1-u_{1}\right) \lambda S_{a}+\left(1-u_{2}\right) \rho I_{n}-(\mu+v+\phi) I_{a} .
\end{gathered}
$$

The parameters $B_{1}, B_{2}$, and $B_{3}$ represent the weight constants of the susceptible substance abusers, the infectious nonsubstance abusers, and the infectious substance abusers, while 
the parameters $A_{1}$ and $A_{2}$ are the weights and cost of condom use and educational campaigns for the controls $u_{1}$ and $u_{2}$, respectively. The terms $u_{1}^{2}$ and $u_{2}^{2}$ reflect the effectiveness of condom use and educational campaigns in the control of the disease. The values $u_{1}=u_{2}=1$ represent maximum effectiveness of condom use and educational campaigns. We therefore seek an optimal control $u_{1}^{*}$ and $u_{2}^{*}$ such that

$$
J\left(u_{1}^{*}, u_{2}^{*}\right)=\max \left\{J\left(u_{1}, u_{2}\right) \mid u_{1}, u_{2} \in \mathcal{U}\right\},
$$

where $\mathscr{U}=\left\{u_{1}(t), u_{2}(t) \mid u_{1}(t), u_{2}(t)\right.$ is measurable: $0 \leq a_{11} \leq$ $\left.u_{1}(t) \leq b_{11} \leq 1,0 \leq a_{22} \leq u_{2}(t) \leq b_{22} \leq 1, t \in\left[0, t_{f}\right]\right\}$.

The basic framework of this problem is to characterize the optimal control and prove the existence of the optimal control and uniqueness of the optimality system.

In our analysis, we assume $\Lambda=\mu N, v=0$, as applied in [56]. Thus the total population $N$ is constant. We can also treat the nonconstant population case by these same techniques, but we choose to present the constant population case here.

5.1. Existence of an Optimal Control. The existence of an optimal control is proved by a result from Fleming and Rishel (1975) [57]. The boundedness of solutions of system (39) for a finite interval is used to prove the existence of an optimal control. To determine existence of an optimal control to our problem, we use a result from Fleming and Rishel (1975) (Theorem 4.1 pages 68-69), where the following properties are satisfied.

(1) The class of all initial conditions with an optimal control set $u_{1}$ and $u_{2}$ in the admissible control set along with each state equation being satisfied is not empty.

(2) The control set $\mathcal{U}$ is convex and closed.

(3) The right-hand side of the state is continuous, is bounded above by a sum of the bounded control and the state, and can be written as a linear function of each control in the optimal control set $u_{1}$ and $u_{2}$ with coefficients depending on time and the state variables.

(4) The integrand of the functional is concave on $\mathcal{U}$ and is bounded above by $c_{2}-c_{1}\left(\left|u_{1}\right|^{2}+\left|u_{2}\right|^{2}\right)$, where $c_{1}, c_{2}>0$.

An existence result in Lukes (1982) [58] (Theorem 9.2.1) for the system of (39) for bounded coefficients is used to give condition 1 . The control set is closed and convex by definition. The right-hand side of the state system equation (39) satisfies condition 3 since the state solutions are a priori bounded. The integrand in the objective functional, $S_{n}-B_{1} S_{a}-B_{2} I_{n}-$ $B_{3} I_{a}-\left(A_{1} u_{1}^{2}+A_{2} u_{2}^{2}\right)$, is Lebesgue integrable and concave on $\mathcal{U}$. Furthermore, $c_{1}, c_{2}>0$ and $B_{1}, B_{2}, B_{3}, A_{1}, A_{2}>1$, hence satisfying

$$
\begin{aligned}
S_{n} & -B_{1} S_{a}-B_{2} I_{n}-B_{3} I_{a}-\left(A_{1} u_{1}^{2}+A_{2} u_{2}^{2}\right) \\
& \leq c_{2}-c_{1}\left(\left|u_{1}\right|^{2}+\left|u_{2}\right|^{2}\right) ;
\end{aligned}
$$

hence the optimal control exists, since the states are bounded.
5.2. Characterisation. Since there exists an optimal control for maximising the functional (42) subject to (39), we use Pontryagin's maximum principle to derive the necessary conditions for this optimal control. The Lagrangian is defined as

$$
\begin{aligned}
& L=S_{n}-B_{1} S_{a}-B_{2} I_{n}-B_{3} I_{a}-\left(A_{1} u_{1}^{2}+A_{2} u_{2}^{2}\right) \\
& +\lambda_{1}\left[\pi_{0} \Lambda-\frac{\alpha\left(1-u_{1}\right) \beta I_{a} S_{n}}{N}\right. \\
& -\frac{\alpha\left(1-u_{1}\right)(1-\eta) \beta I_{n} S_{n}}{N} \\
& \left.+\phi S_{a}-\left(1-u_{2}\right) \rho S_{n}-\mu S_{n}\right] \\
& +\lambda_{2}\left[\left(1-u_{2}\right) \pi_{1} \Lambda-\frac{\left(1-u_{1}\right) \beta I_{a} S_{a}}{N}\right. \\
& -\frac{\left(1-u_{1}\right)(1-\eta) \beta I_{n} S_{a}}{N} \\
& \left.+\left(1-u_{2}\right) \rho S_{n}-(\mu+v+\phi) S_{a}\right] \\
& +\lambda_{3}\left[\frac{\alpha\left(1-u_{1}\right) \beta I_{a} S_{n}}{N}\right. \\
& +\frac{\alpha\left(1-u_{1}\right)(1-\eta) \beta I_{n} S_{n}}{N} \\
& \left.+\phi I_{a}-\left(1-u_{2}\right) \rho I_{n}-\mu I_{n}\right] \\
& +\lambda_{4}\left[\frac{\left(1-u_{1}\right) \beta I_{a} S_{a}}{N}+\frac{\left(1-u_{1}\right)(1-\eta) \beta I_{n} S_{a}}{N}\right. \\
& \left.+\left(1-u_{2}\right) \rho I_{n}-(\mu+v+\phi) I_{a}\right] \\
& +\omega_{11}(t)\left(b_{1}-u_{1}(t)\right)+\omega_{12}(t)\left(u_{1}(t)-a_{1}\right) \\
& +\omega_{21}(t)\left(b_{2}-u_{2}(t)\right)+\omega_{22}(t)\left(u_{2}(t)-a_{2}\right),
\end{aligned}
$$

where $\omega_{11}(t), \omega_{12}(t) \geq 0, \omega_{21}(t)$, and $\omega_{22}(t) \geq 0$ are penalty multipliers satisfying $\omega_{11}(t)\left(b_{1}-u_{1}(t)\right)=0, \omega_{12}(t)\left(u_{1}(t)-\right.$ $\left.a_{1}\right)=0, \omega_{21}(t)\left(b_{2}-u_{2}(t)\right)=0$ and $\omega_{22}(t)\left(u_{2}(t)-a_{2}\right)=0$, at the optimal point $u_{1}^{*}$ and $u_{2}^{*}$.

Theorem 9. Given optimal controls $u_{1}^{*}$ and $u_{2}^{*}$ and solutions of the corresponding state system (39), there exist adjoint variables $\lambda_{i}, i=1, \ldots, 4$ satisfying

$$
\begin{aligned}
\frac{d \lambda_{1}}{d t}= & -\frac{\partial L}{\partial S_{n}} \\
= & -1+\frac{\alpha\left(1-u_{1}\right) \beta I_{a}\left[\lambda_{1}-\lambda_{3}\right]}{N} \\
& +\frac{\alpha\left(1-u_{1}\right)(1-\eta) \beta I_{n}\left[\lambda_{1}-\lambda_{3}\right]}{N} \\
& +\left(1-u_{2}\right) \rho\left[\lambda_{1}-\lambda_{2}\right]+\mu \lambda_{1},
\end{aligned}
$$




$$
\begin{aligned}
\frac{d \lambda_{2}}{d t}= & -\frac{\partial L}{\partial S_{a}} \\
= & B_{1}+\phi\left[\lambda_{2}-\lambda_{1}\right]+\frac{\left(1-u_{1}\right) \beta I_{a}\left[\lambda_{2}-\lambda_{4}\right]}{N} \\
& +\frac{\left(1-u_{1}\right)(1-\eta) \beta I_{n}\left[\lambda_{2}-\lambda_{4}\right]}{N}+(\mu+v) \lambda_{2} \\
\frac{d \lambda_{2}}{d t=} & -\frac{\partial L}{\partial I_{n}} \\
= & B_{2}+\frac{\alpha\left(1-u_{1}\right)(1-\eta) \beta S_{n}\left[\lambda_{1}-\lambda_{3}\right]}{N} \\
& +\frac{\left(1-u_{1}\right)(1-\eta) \beta S_{a}\left[\lambda_{2}-\lambda_{4}\right]}{N} \\
& +\left(1-u_{2}\right) \rho\left[\lambda_{3}-\lambda_{4}\right]+\mu \lambda_{3}, \\
\frac{d \lambda_{2}}{d t=} & -\frac{\partial L}{\partial I_{n}} \\
= & B_{3}+\frac{\alpha\left(1-u_{1}\right) \beta S_{n}\left[\lambda_{1}-\lambda_{3}\right]}{N} \\
& \left(1-u_{1}\right) \beta S_{a}\left[\lambda_{2}-\lambda_{4}\right] \\
N & \frac{\alpha\left[\lambda_{4}-\lambda_{3}\right]+(\mu+v) \lambda_{4}}{N}
\end{aligned}
$$

Proof. The form of the adjoint equation and transversality conditions are standard results from Pontryagin's maximum principle $[57,59]$; therefore, solutions to the adjoint system exist and are bounded. To determine the interior maximum of our Lagrangian, we take the partial derivative $L$ with respect to $u_{1}(t)$ and $u_{2}(t)$ and set to zero. Thus, we have the following.

$u_{1}(t)^{*}$ is subject of formulae

$$
\begin{aligned}
u_{1}(t)^{*}= & \frac{\beta\left(I_{a}+(1-\eta) I_{n}\right)}{2 A_{1} N}\left[\alpha S_{n}\left(\lambda_{1}-\lambda_{3}\right)+S_{a}\left(\lambda_{2}-\lambda_{4}\right)\right] \\
& +\frac{\omega_{12}(t)-\omega_{11}(t)}{2 A_{1}} .
\end{aligned}
$$

$u_{2}(t)^{*}$ is subject of formulae

$$
\begin{aligned}
u_{2}(t)^{*}= & \frac{\rho}{2 A_{2}}\left[S_{n}\left(\lambda_{1}-\lambda_{2}\right)+I_{n}\left(\lambda_{3}-\lambda_{4}\right)\right]-\frac{\pi_{1} \Lambda \lambda_{2}}{2 A_{2}} \\
& +\frac{\omega_{22}-\omega_{21}}{2 A_{2}} .
\end{aligned}
$$

To determine the explicit expression for the control without $\omega_{1}(t)$ and $\omega_{2}(t)$, a standard optimality technique is utilised. The specific characterisation of the optimal controls $u_{1}(t)^{*}$ and $u_{2}(t)^{*}$. Consider

$$
\begin{aligned}
& u_{1}(t)^{*} \\
& =\min \left\{\operatorname { m a x } \left\{a_{1}, \frac{\beta\left(I_{a}+(1-\eta) I_{n}\right)}{2 A_{1} N}\right.\right. \\
& \left.\left.\times\left[\alpha S_{n}\left(\lambda_{1}-\lambda_{3}\right)+S_{a}\left(\lambda_{2}-\lambda_{4}\right)\right]\right\}, b_{1}\right\} \\
& u_{2}(t)^{*} \\
& =\min \left\{\operatorname { m a x } \left\{a_{2}, \frac{\rho}{2 A_{2}}\left[S_{n}\left(\lambda_{1}-\lambda_{2}\right)+I_{n}\left(\lambda_{3}-\lambda_{4}\right)\right]\right.\right. \\
& \left.\left.-\frac{\pi_{1} \Lambda \lambda_{2}}{2 A_{2}}\right\}, b_{2}\right\} \text {. }
\end{aligned}
$$

The optimality system consists of the state system coupled with the adjoint system with the initial conditions, the transversality conditions and the characterisation of the optimal control. Substituting $u_{1}^{*}$, and $u_{2}^{*}$ for $u_{1}(t)$, and $u_{2}(t)$, in (43) gives the optimality system. The state system and adjoint system have finite upper bounds. These bounds are needed in the uniqueness proof of the optimality system. Due to a priori boundedness of the state and adjoint functions and the resulting Lipschitz structure of the ODEs, we obtain the uniqueness of the optimal control for small $t_{f}$ [60]. The uniqueness of the optimal control follows from the uniqueness of the optimality system.

5.3. Numerical Simulations. The optimality system is solved using an iterative method with Runge-Kutta fourth-order scheme. Starting with a guess for the adjoint variables, the state equations are solved forward in time. Then these state values are used to solve the adjoint equations backward in time, and the iterations continue until convergence. The simulations were carried out using parameter values in Table 1 and the following are the values $A_{1}=0.005, A_{2}=$ $0.005, B_{1}=0.06, B_{2}=0.05$, and $B_{3}=0.05$. The assumed initial conditions for the differential equations are $S_{n_{0}}=0.4$, $S_{a_{0}}=0.3, I_{n_{0}}=0.2$, and $I_{a_{0}}=0.1$.

Figure 8(a) illustrates the population of the susceptible nonsubstance abusing adolescents in the presence and absence of the controls over a period of 20 years. For the period 0-3 years, in the absence of the control, the susceptible nonsubstance abusing adolescents have a sharp increase, and for the same period when there is no control the population of the nonsubstance abusing adolescents decreases sharply. Further, we note that for the case when there is no control, for the period 3-20 years, the population of the susceptible nonsubstance abusing adolescents increases slightly.

Figure 8(b) illustrates the population of the susceptible substance abusers adolescents in the presence and absence of the controls over a period of 20 years. For the period $0-3$ years, in the presence of the controls, the susceptible substance abusing adolescents have a sharp decrease, and for the same period when there are controls the population of 


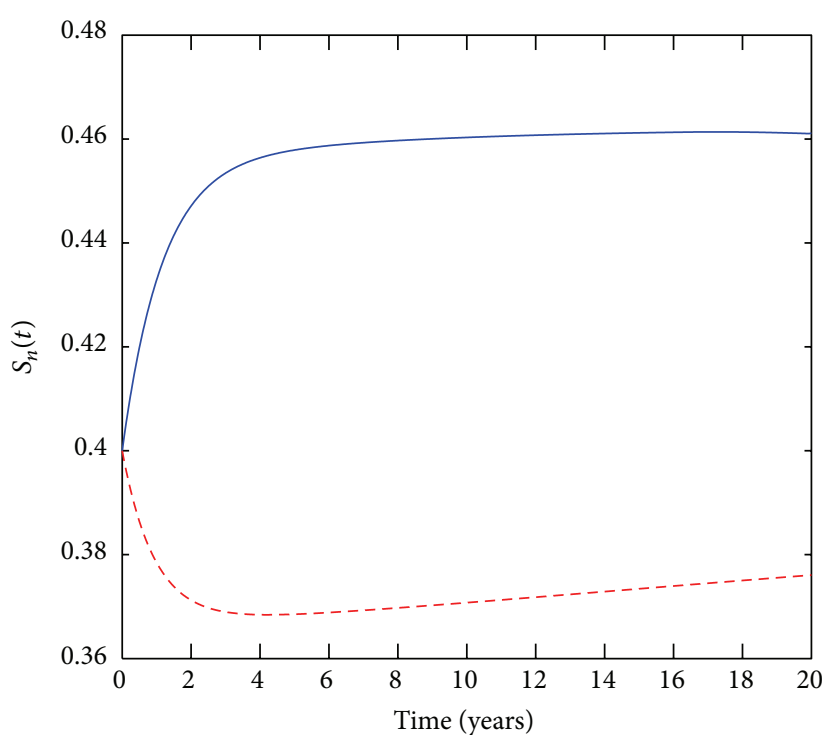

(a)

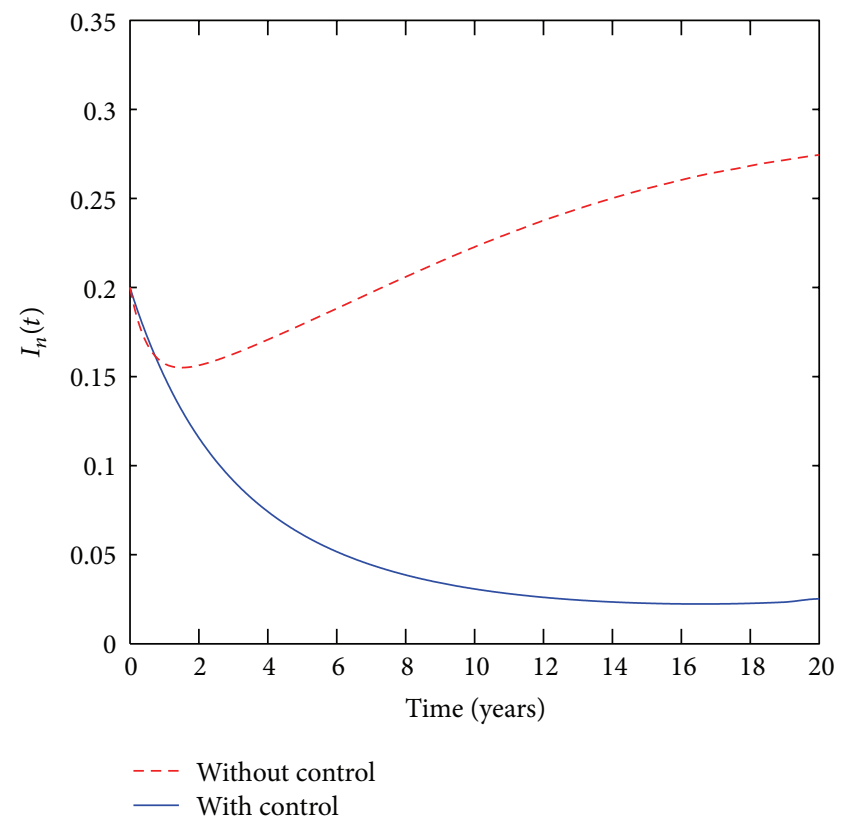

(c)

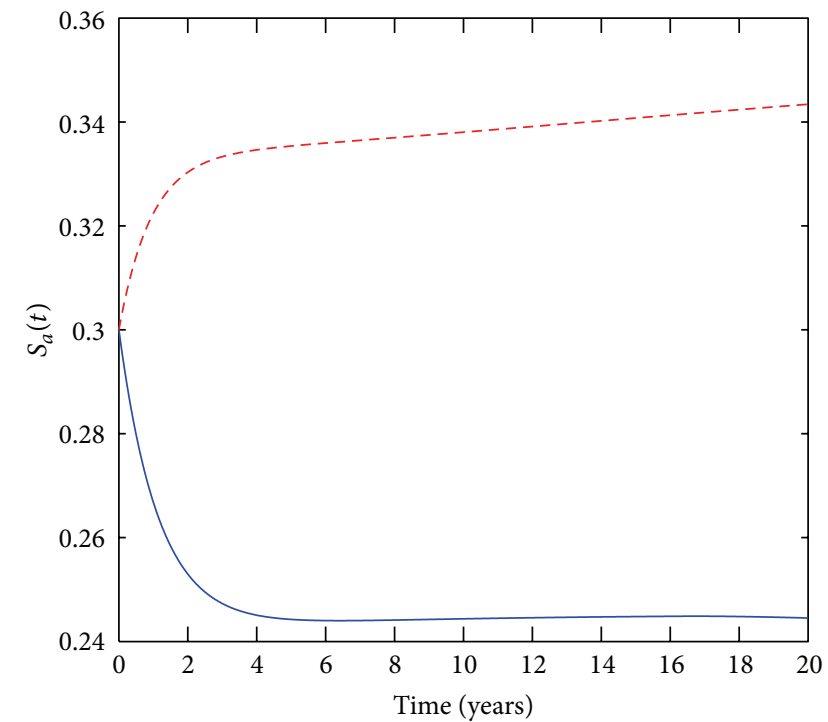

(b)

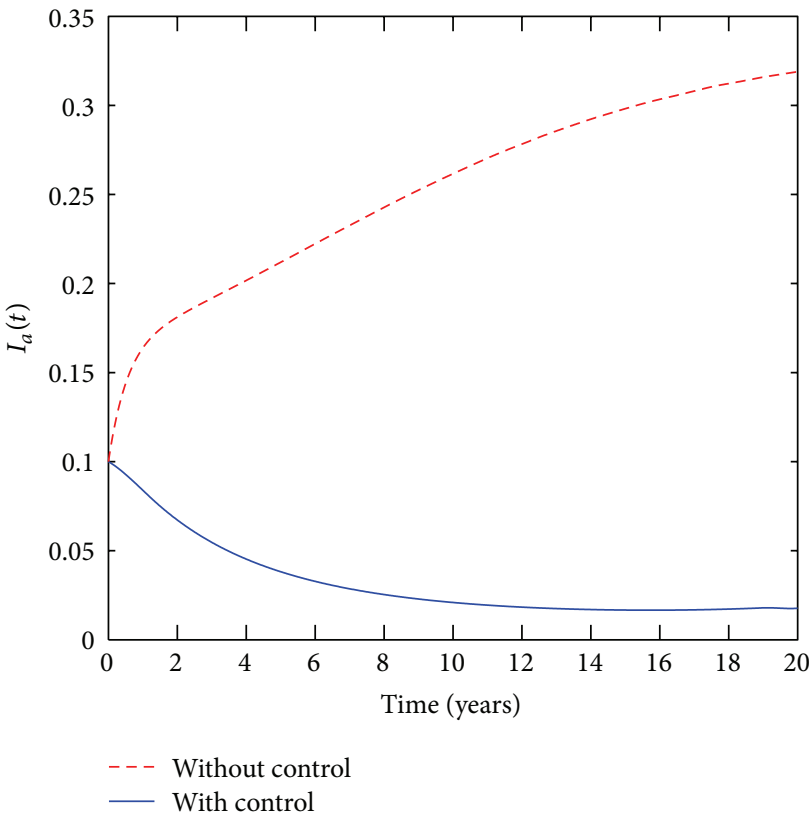

(d)

Figure 8: Time series plots showing the effects of optimal control on the susceptible nonsubstance abusing adolescents $S_{n}$, susceptible substance abusing adolescents $S_{a}$, infectious nonsubstance abusing adolescents $I_{n}$, and the infectious substance abusing $I_{a}$, over a period of 20 years.

the susceptible substance abusers increases sharply. It is worth noting that, after 3 years, the population for the substance abusers with control remains constant while the population of the susceptible substance abusers without control continues increasing gradually.

Figure 8(c) highlights the impact of controls on the population of infectious nonsubstance abusing adolescents. For the period 0-2 years, in the absence of the control, cumulative HSV-2 cases for the nonsubstance abusing adolescents decrease rapidly, before starting to increase moderately for the remaining years under consideration. The cumulative HSV-2 cases for the nonsubstance abusing adolescents decrease sharply for the whole 20 years under consideration. It is worth noting that, for the infectious nonsubstance abusing adolescents, the controls yield positive results after a period of 2 years; hence the controls have an impact in controlling cumulative HSV-2 cases, within a community.

Figure 8(d) highlights the impact of controls on the population of infectious substance abusing adolescents. For the whole period under investigation, in the absence of the control, cumulative infectious substance abusing HSV2 cases increase rapidly, and, for the similar period, when there is a control the population of the exposed individuals decreases sharply. Results on Figure 8(d) clearly suggest that 


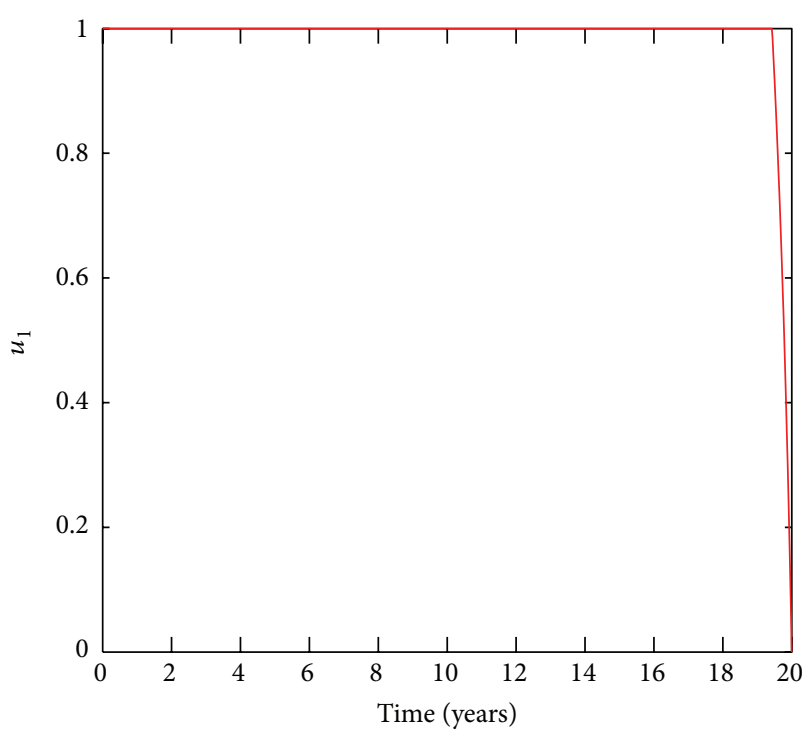

(a)

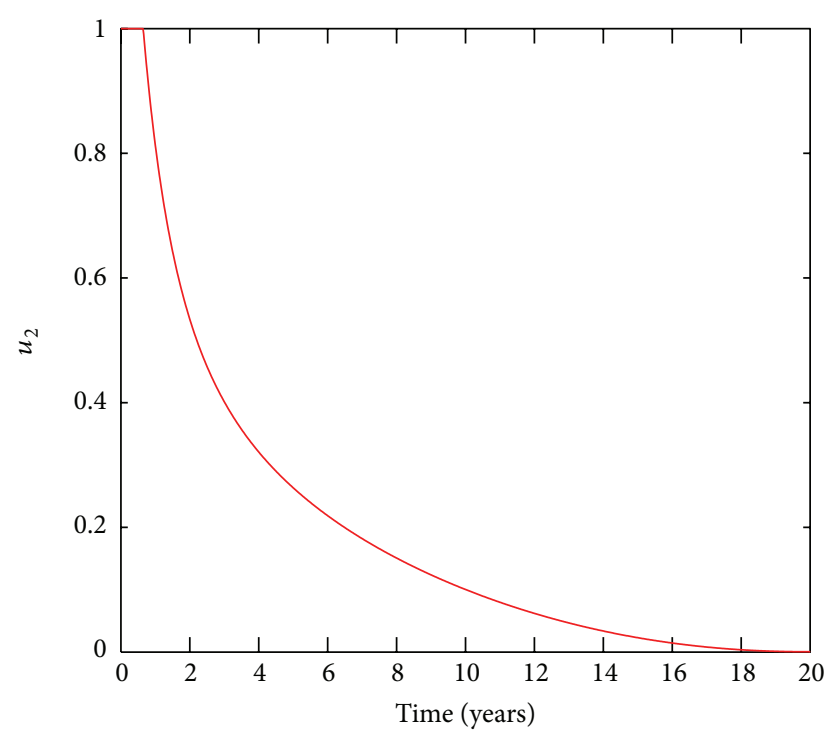

(b)

FIGURE 9: The optimal control graphs for the two controls, namely, condom use $\left(u_{1}\right)$ and educational campaigns $\left(u_{2}\right)$.

the presence of controls has an impact on reducing cumulative HSV-2 cases within a society with substance abusing adolescents.

Figure 9 represents the controls $u_{1}$ and $u_{2}$. The condom use control $u_{1}$ is at the upper bound $b_{1}$ for approximately 20 years and has a steady drop until it reaches the lower bound $a_{1}=0$. Treatment control, $u_{2}$, is at the upper bound for approximately half a year and has a sharp drop until it reaches the lower bound $a_{2}=0$.

These results suggest that more effort should be devoted to condom use $\left(u_{1}\right)$, as compared to educational campaigns $\left(u_{2}\right)$.

\section{Discussion}

Adolescents who abuse substances have higher rates of HSV2 infection, since adolescents who engage in substance abuse also engage in risky behaviours; hence most of them do not use condoms. In general adolescents are not fully educated on HSV-2. A mathematical model for investigating the effects of substance abuse amongst adolescents on the transmission dynamics of HSV-2 in the community is formulated and analysed. We computed and compared the reproduction numbers. Results from the analysis of the reproduction numbers suggest that condom use and educational campaigns have a great impact in the reduction of HSV-2 cases in the community. The PRCCs were calculated to estimate the correlation between values of the reproduction number and the epidemiological parameters. It has been seen that an increase in condom use and a reduction on the number of adolescents becoming substance abusers have the greatest influence on reducing the magnitude of the reproduction number than any other epidemiological parameters (Figure 6). This result is further supported by numerical simulations which show that condom use is highly effective when we are having more adolescents quitting substance abusing habit than those becoming ones, whereas it is less effective when we have more adolescents becoming substance abusers than quitting. Substance abuse amongst adolescents increases disease transmission, and prevalence of disease increases with increased rate of becoming a substance abuser. Thus, in the event when we have more individuals becoming heavy substance abusers, there is urgent need for intervention strategies such as counselling and educational campaigns in order to curtail the HSV-2 spread. Numerical simulations also show and suggest that an increase in recruitment of substance abusers in the community will increase the prevalence of HSV-2 cases.

The optimal control results show how a cost effective combination of aforementioned HSV-2 intervention strategies (condom use and educational campaigns) amongst adolescents may influence cumulative cases over a period of 20 years. Overall, optimal control theory results suggest that more effort should be devoted to condom use compared to educational campaigns.

\section{Appendices}

\section{A. Positivity of $H$}

To show that $H \geq 0$, it is sufficient to show that

$$
\begin{aligned}
& \frac{\pi_{0}(\mu+v)+\phi}{\mu+\phi+\rho+v \pi_{0}} \geq \frac{S_{n}}{N}, \\
& \frac{\mu \pi_{1}+\rho}{\mu+\phi+\rho+v \pi_{0}} \geq \frac{S_{a}}{N} .
\end{aligned}
$$


To do this we prove it by contradiction. Assume the following statements are true:

$$
\begin{aligned}
& \text { (i) } \frac{\pi_{0}(\mu+v)+\phi}{\mu+\phi+\rho+v \pi_{0}}<\frac{S_{n}}{N}, \\
& \text { (ii) } \frac{\mu \pi_{1}+\rho}{\mu+\phi+\rho+v \pi_{0}}<\frac{S_{a}}{N} .
\end{aligned}
$$

Adding the inequalities (i) and (ii) in (A.2), we have that

$$
\frac{\mu\left(\pi_{0}+\pi_{1}\right)+\phi+\rho+\pi_{0}}{\mu+\phi+\rho+v \pi_{0}}<\frac{S_{a}+S_{n}}{N} \Longrightarrow 1<1,
$$

which is not true, hence a contradiction (since a number cannot be less than itself).

Equations (A.2) and (A.5) should justify that $H \geq 0$. In order to prove this we shall use the direct proof; for example, if

$$
\begin{aligned}
& \text { (i) } H_{1} \geq 2 \text {, } \\
& \text { (ii) } H_{2} \geq 1 \text {. }
\end{aligned}
$$

Adding items (i) and (ii) one gets $H_{1}+H_{2} \geq 3$.

Using the above argument $H \geq 0$ if and only if

$$
\begin{aligned}
& \text { (i) } \frac{\pi_{0}(\mu+v)+\phi}{\mu+\phi+\rho+v \pi_{0}} \geq \frac{S_{n}}{N}, \\
& \text { (ii) } \frac{\mu \pi_{1}+\rho}{\mu+\phi+\rho+v \pi_{0}} \geq \frac{S_{a}}{N} .
\end{aligned}
$$

Adding items (i) and (ii) one gets

$$
\begin{aligned}
\frac{\mu\left(\pi_{0}+\pi_{1}\right)+\phi+\rho+v \pi_{0}}{\mu+\phi+\rho+v \pi_{0}} & \geq \frac{S_{a}+S_{n}}{N} \\
& \Longrightarrow \frac{\mu+\phi+\rho+v \pi_{0}}{\mu+\phi+\rho+v \pi_{0}} \\
& \geq \frac{S_{a}+S_{n}}{N} \Longrightarrow 1 \geq 1 .
\end{aligned}
$$

Thus, from the above argument $H \geq 0$.

\section{B. Poincaré-Bendixson's Negative Criterion}

Consider the nonlinear autonomous system

$$
x^{\prime}=f(x),
$$

where $f \in C^{1}(\mathbb{R} \rightarrow \mathbb{R})$. If $x_{0} \in \mathbb{R}^{n}$, let $x=x\left(t, x_{0}\right)$ be the solution of (B.1) which satisfies $x\left(0, x_{0}\right)=x_{0}$. Bendixon's negative criterion: when $n=2$, a sufficient condition for the nonexistence of nonconstant periodic solutions of (B.1) is that, for each $x \in \mathbb{R}^{2}, \operatorname{div} f(x) \neq 0$.

Theorem B.1. Suppose that one of the inequalities $\mu\left(\partial f^{[2]} / \partial x\right)<0, \mu\left(-\partial f^{[2]} / \partial x\right)<0$, holds for all $x \in \mathbb{R}^{n}$. Then the system (B.1) has nonconstant periodic solutions.

For a detailed discussion of the Bendixon's negative criterion, we refer the reader to $[51,61]$.

\section{Conflict of Interests}

The authors declare that there is no conflict of interests regarding the publication of this paper.

\section{Acknowledgments}

The authors would like to thank the handling editor and anonymous reviewers for their comments and suggestions which improved the paper.

\section{References}

[1] K. J. Looker, G. P. Garnett, and G. P. Schmid, "An estimate of the global prevalence and incidence of herpes simplex virus type 2 infection," Bulletin of the World Health Organization, vol. 86, no. 10, pp. 805A-812A, 2008.

[2] K. J. Looker, G. P. Garnett, and G. P. Schmid, "An estimate of the global prevalence and incidence of herpes simplex virus type 2 infection," Bulletin of the World Health Organization, vol. 86, no. 10, pp. 805-812, 2008.

[3] CDC [Centers for Disease Control and Prevention], Division of Sexually Transmitted Disease Surveillance 1999. Atlanta: Department of Health and Human Services, Centers for Disease Control and Prevention (CDC), 2000.

[4] K. Owusu-Edusei Jr., H. W. Chesson, T. L. Gift et al., "The estimated direct medical cost of selected sexually transmitted infections in the United States, 2008," Sexually Transmitted Diseases, vol. 40, no. 3, pp. 197-201, 2013.

[5] WHO [World Health Organisation], The Second Decade: Improving Adolescent Health and Development, WHO Adolescent and Development Programme (WHO/FRH/ADH/98.19), Geneva, Switzerland, 1998.

[6] K. L. Dehne and G. Riedner, Sexually Transmitted Infections among Adolescents: The Need for Adequate Health Care, WHO, Geneva, Switzerland, 2005.

[7] Y. L. Hill and F. M. Biro, Adolescents and Sexually Transmitted Infections, CME Feature, 2009.

[8] CDC [Centers for Disease Control and Prevention] Division of STD Prevention, Sexually Transmitted Disease Surveillance 1999, Department of Health and Human Services, Centers for Disease Control and Prevention (CDC), Atlanta, Ga, USA, 2000.

[9] W. Cates and M. C. Pheeters, "Adolescents and sexually transmitted diseases: current risks and future consequences," in Proceedings of the Workshop on Adolescent Sexuality and Reproductive Health in Developing Countries: Trends and Interventions, National Research Council, Washington, DC, USA, March 1997.

[10] K. C. Chinsembu, "Sexually transmitted infections in adolescents," The Open Infectious Diseases Journal, vol. 3, pp. 107-117, 2009.

[11] S. Y. Cheng and K. K. Lo, "Sexually transmitted infections in adolescents," Hong Kong Journal of Paediatrics, vol. 7, no. 2, pp. 76-84, 2002.

[12] S. L. Rosenthal, L. R. Stanberry, F. M. Biro et al., "Seroprevalence of herpes simplex virus types 1 and 2 and cytomegalovirus in adolescents," Clinical Infectious Diseases, vol. 24, no. 2, pp. 135139, 1997. 
[13] G. Sucato, C. Celum, D. Dithmer, R. Ashley, and A. Wald, "Demographic rather than behavioral risk factors predict herpes simplex virus type 2 infection in sexually active adolescents," Pediatric Infectious Disease Journal, vol. 20, no. 4, pp. 422-426, 2001.

[14] J. Noell, P. Rohde, L. Ochs et al., "Incidence and prevalence of Chlamydia, herpes, and viral hepatitis in a homeless adolescent population," Sexually Transmitted Diseases, vol. 28, no. 1, pp. 4$10,2001$.

[15] R. L. Cook, N. K. Pollock, A. K. Rao, and D. B. Clark, "Increased prevalence of herpes simplex virus type 2 among adolescent women with alcohol use disorders," Journal of Adolescent Health, vol. 30, no. 3, pp. 169-174, 2002.

[16] C. D. Abraham, C. J. Conde-Glez, A. Cruz-Valdez, L. SánchezZamorano, C. Hernández-Márquez, and E. Lazcano-Ponce, "Sexual and demographic risk factors for herpes simplex virus type 2 according to schooling level among mexican youths," Sexually Transmitted Diseases, vol. 30, no. 7, pp. 549-555, 2003.

[17] I. J. Birdthistle, S. Floyd, A. MacHingura, N. Mudziwapasi, S. Gregson, and J. R. Glynn, "From affected to infected? Orphanhood and HIV risk among female adolescents in urban Zimbabwe," AIDS, vol. 22, no. 6, pp. 759-766, 2008.

[18] P. N. Amornkul, H. Vandenhoudt, P. Nasokho et al., "HIV prevalence and associated risk factors among individuals aged 13-34 years in rural Western Kenya," PLoS ONE, vol. 4, no. 7, Article ID e6470, 2009.

[19] Centers for Disease Control and Prevention, "Youth risk behaviour surveillance-United States," Morbidity and Mortality Weekly Report, vol. 53, pp. 1-100, 2004.

[20] K. Buchacz, W. McFarland, M. Hernandez et al., "Prevalence and correlates of herpes simplex virus type 2 infection in a population-based survey of young women in low-income neighborhoods of Northern California," Sexually Transmitted Diseases, vol. 27, no. 7, pp. 393-400, 2000.

[21] L. Corey, A. Wald, C. L. Celum, and T. C. Quinn, "The effects of herpes simplex virus-2 on HIV-1 acquisition and transmission: a review of two overlapping epidemics," Journal of Acquired Immune Deficiency Syndromes, vol. 35, no. 5, pp. 435-445, 2004.

[22] E. E. Freeman, H. A. Weiss, J. R. Glynn, P. L. Cross, J. A. Whitworth, and R. J. Hayes, "Herpes simplex virus 2 infection increases HIV acquisition in men and women: systematic review and meta-analysis of longitudinal studies," AIDS, vol. 20, no. 1, pp. 73-83, 2006.

[23] S. Stagno and R. J. Whitley, "Herpes virus infections in neonates and children: cytomegalovirus and herpes simplex virus," in Sexually Transmitted Diseases, K. K. Holmes, P. F. Sparling, P. M. Mardh et al., Eds., pp. 1191-1212, McGraw-Hill, New York, NY, USA, 3rd edition, 1999.

[24] A. Nahmias, W. E. Josey, Z. M. Naib, M. G. Freeman, R. J. Fernandez, and J. H. Wheeler, "Perinatal risk associated with maternal genital herpes simplex virus infection," The American Journal of Obstetrics and Gynecology, vol. 110, no. 6, pp. 825-837, 1971.

[25] C. Johnston, D. M. Koelle, and A. Wald, "Current status and prospects for development of an HSV vaccine," Vaccine, vol. 32, no. 14, pp. 1553-1560, 2014.

[26] S. D. Moretlwe and C. Celum, "HSV-2 treatment interventions to control HIV: hope for the future," HIV Prevention, Counselling and Testing, pp. 649-658, 2004.

[27] C. P. Bhunu and S. Mushayabasa, "Assessing the effects of intravenous drug use on hepatitis C transmission dynamics," Journal of Biological Systems, vol. 19, no. 3, pp. 447-460, 2011.
[28] C. P. Bhunu and S. Mushayabasa, "Prostitution and drug (alcohol) misuse: the menacing combination," Journal of Biological Systems, vol. 20, no. 2, pp. 177-193, 2012.

[29] Y. J. Bryson, M. Dillon, D. I. Bernstein, J. Radolf, P. Zakowski, and E. Garratty, "Risk of acquisition of genital herpes simplex virus type 2 in sex partners of persons with genital herpes: a prospective couple study," Journal of Infectious Diseases, vol. 167, no. 4, pp. 942-946, 1993.

[30] L. Corey, A. Wald, R. Patel et al., "Once-daily valacyclovir to reduce the risk of transmission of genital herpes," The New England Journal of Medicine, vol. 350, no. 1, pp. 11-20, 2004.

[31] G. J. Mertz, R. W. Coombs, R. Ashley et al., "Transmission of genital herpes in couples with one symptomatic and one asymptomatic partner: a prospective study," The Journal of Infectious Diseases, vol. 157, no. 6, pp. 1169-1177, 1988.

[32] A. Wald, A. G. Langenberg, K. Link et al., "Effect of condoms on reducing the transmission of herpes simplex virus type 2 from men to women," Journal of the American Medical Association, vol. 285, no. 24, pp. 3100-3106, 2001.

[33] A. H. Mokdad, J. S. Marks, D. F. Stroup, and J. L. Gerberding, "Actual causes of death in the United States, 2000," The Journal of the American Medical Association, vol. 291, no. 10, pp. 1238$1245,2004$.

[34] A. Wald, A. G. Langenberg, K. Link et al., "Effect of condoms on reducing the transmission of herpes simplex virus type 2 from men to women," Journal of the American Medical Association, vol. 285, no. 24, pp. 3100-3106, 2001.

[35] E. T. Martin, E. Krantz, S. L. Gottlieb et al., "A pooled analysis of the effect of condoms in preventing HSV-2 acquisition," Archives of Internal Medicine, vol. 169, no. 13, pp. 1233-1240, 2009.

[36] A. C. Ghani and S. O. Aral, "Patterns of sex worker-client contacts and their implications for the persistence of sexually transmitted infections," Journal of Infectious Diseases, vol. 191, no. 1, pp. S34-S41, 2005.

[37] G. P. Garnett, G. Dubin, M. Slaoui, and T. Darcis, "The potential epidemiological impact of a genital herpes vaccine for women," Sexually Transmitted Infections, vol. 80, no. 1, pp. 24-29, 2004.

[38] E. J. Schwartz and S. Blower, "Predicting the potential individual- and population-level effects of imperfect herpes simplex virus type 2 vaccines," The Journal of Infectious Diseases, vol. 191, no. 10, pp. 1734-1746, 2005.

[39] C. N. Podder and A. B. Gumel, "Qualitative dynamics of a vaccination model for HSV-2," IMA Journal of Applied Mathematics, vol. 75, no. 1, pp. 75-107, 2010.

[40] R. M. Alsallaq, J. T. Schiffer, I. M. Longini, A. Wald, L. Corey, and L. J. Abu-Raddad, "Population level impact of an imperfect prophylactic vaccine," Sexually Transmitted Diseases, vol. 37, no. 5, pp. 290-297, 2010.

[41] Y. Lou, R. Qesmi, Q. Wang, M. Steben, J. Wu, and J. M. Heffernan, "Epidemiological impact of a genital herpes type 2 vaccine for young females," PLoS ONE, vol. 7, no. 10, Article ID e46027, 2012.

[42] A. M. Foss, P. T. Vickerman, Z. Chalabi, P. Mayaud, M. Alary, and C. H. Watts, "Dynamic modeling of herpes simplex virus type-2 (HSV-2) transmission: issues in structural uncertainty," Bulletin of Mathematical Biology, vol. 71, no. 3, pp. 720-749, 2009.

[43] A. M. Geretti, "Genital herpes," Sexually Transmitted Infections, vol. 82, no. 4, pp. iv31-iv34, 2006.

[44] R. Gupta, T. Warren, and A. Wald, "Genital herpes," The Lancet, vol. 370, no. 9605, pp. 2127-2137, 2007. 
[45] E. Kuntsche, R. Knibbe, G. Gmel, and R. Engels, "Why do young people drink? A review of drinking motives," Clinical Psychology Review, vol. 25, no. 7, pp. 841-861, 2005.

[46] B. A. Auslander, F. M. Biro, and S. L. Rosenthal, "Genital herpes in adolescents," Seminars in Pediatric Infectious Diseases, vol. 16, no. 1, pp. 24-30, 2005.

[47] H. W. Hethcote, "The mathematics of infectious diseases," SIAM Review, vol. 42, no. 4, pp. 599-653, 2000.

[48] P. van den Driessche and J. Watmough, "Reproduction numbers and sub-threshold endemic equilibria for compartmental models of disease transmission," Mathematical Biosciences, vol. 180, no. 1-2, pp. 29-48, 2002.

[49] J. Arino, F. Brauer, P. van den Driessche, J. Watmough, and J. Wu, "A model for influenza with vaccination and antiviral treatment," Journal of Theoretical Biology, vol. 253, no. 1, pp. 118$130,2008$.

[50] V. Lakshmikantham, S. Leela, and A. A. Martynyuk, Stability Analysis of Nonlinear Systems, vol. 125 of Pure and Applied Mathematics: A Series of Monographs and Textbooks, Marcel Dekker, New York, NY, USA, 1989.

[51] L. Perko, Differential Equations and Dynamical Systems, vol. 7 of Texts in Applied Mathematics, Springer, Berlin, Germany, 2000.

[52] A. Korobeinikov and P. K. Maini, "A Lyapunov function and global properties for SIR and SEIR epidemiological models with nonlinear incidence," Mathematical Biosciences and Engineering. $M B E$, vol. 1, no. 1, pp. 57-60, 2004.

[53] A. Korobeinikov and G. C. Wake, "Lyapunov functions and global stability for SIR, SIRS, and SIS epidemiological models," Applied Mathematics Letters, vol. 15, no. 8, pp. 955-960, 2002.

[54] I. Barbalat, "Systéme d'équation différentielle d'oscillation nonlinéaires," Revue Roumaine de Mathématique Pures et Appliquées, vol. 4, pp. 267-270, 1959.

[55] C. C. McCluskey, "Lyapunov functions for tuberculosis models with fast and slow progression," Mathematical Biosciences and Engineering. $M B E$, vol. 3, no. 4, pp. 603-614, 2006.

[56] E. Jung, S. Lenhart, and Z. Feng, "Optimal control of treatments in a two-strain tuberculosis model," Discrete and Continuous Dynamical Systems. Series B. A Journal Bridging Mathematics and Sciences, vol. 2, no. 4, pp. 473-482, 2002.

[57] W. H. Fleming and R. W. Rishel, Deterministic and Stochastic Optimal Control, Springer, New York, NY, USA, 1975.

[58] D. L. Lukes, Differential Equations: Classical to Controlled, Mathematics in Science and Engineering, vol. 162, Academic Press, New York, NY, USA, 1982.

[59] L. S. Pontryagin, V. T. Boltyanskii, R. V. Gamkrelidze, and E. F. Mishchevko, The Mathematical Theory of Optimal Processes, vol. 4, Gordon and Breach Science Publishers, 1985.

[60] G. Magombedze, W. Garira, E. Mwenje, and C. P. Bhunu, "Optimal control for HIV-1 multi-drug therapy," International Journal of Computer Mathematics, vol. 88, no. 2, pp. 314-340, 2011.

[61] M. Fiedler, "Additive compound matrices and an inequality for eigenvalues of symmetric stochastic matrices," Czechoslovak Mathematical Journal, vol. 24(99), pp. 392-402, 1974. 


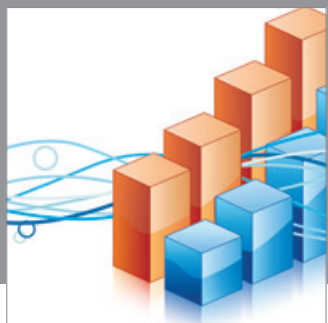

Advances in

Operations Research

mansans

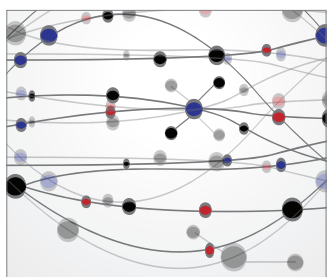

The Scientific World Journal
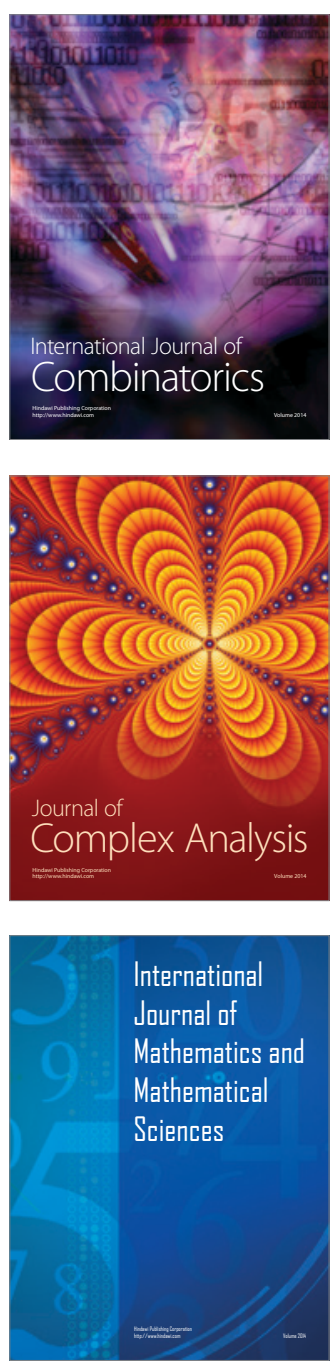
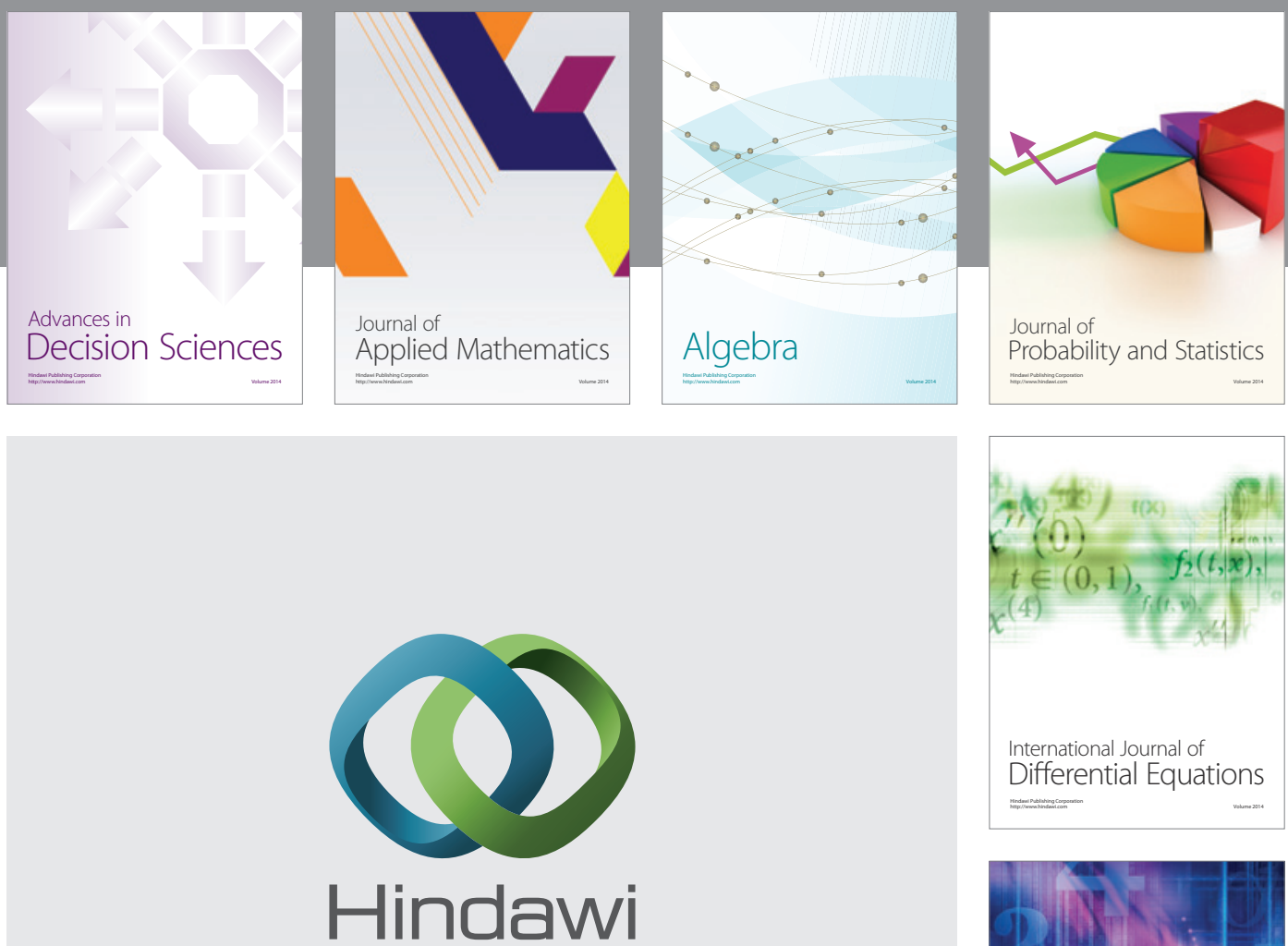

Submit your manuscripts at http://www.hindawi.com
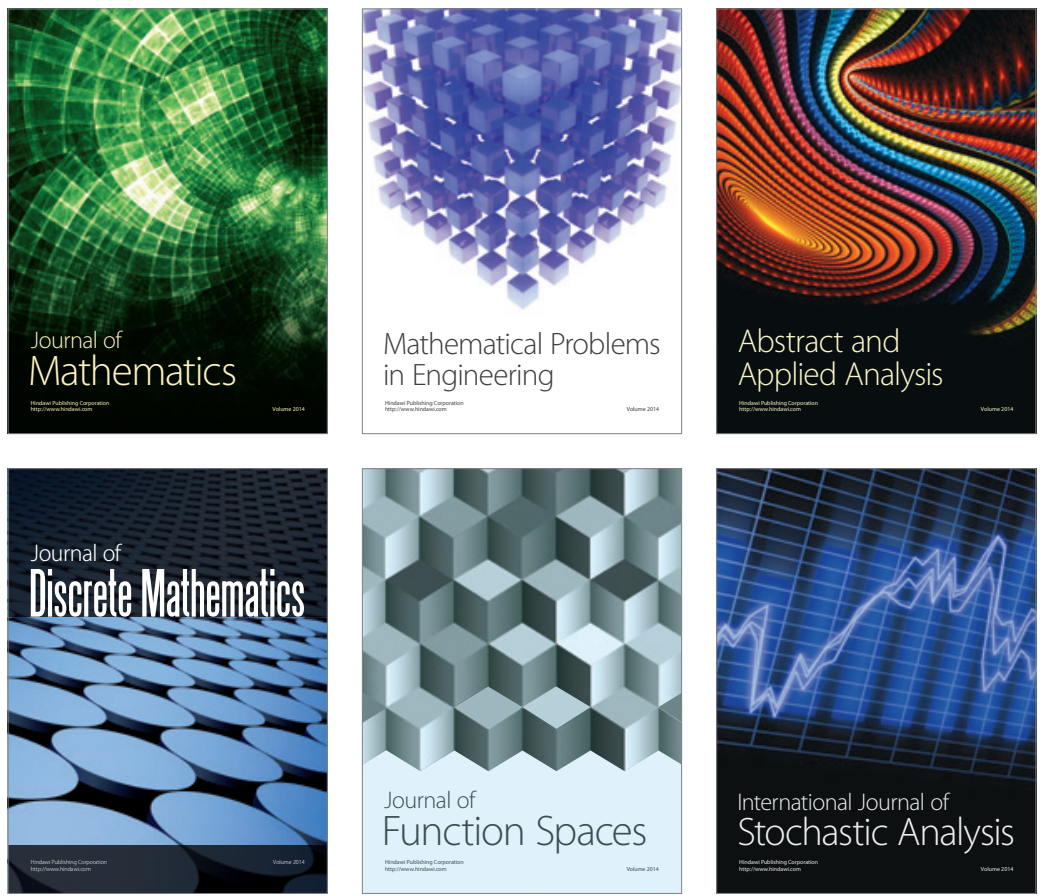

Journal of

Function Spaces

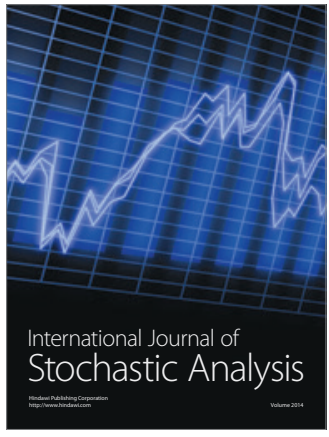

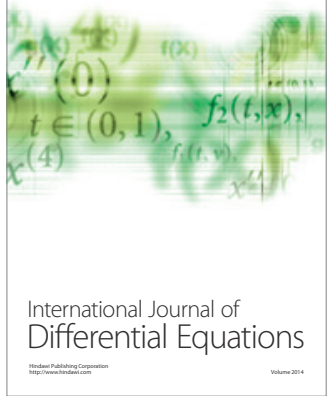
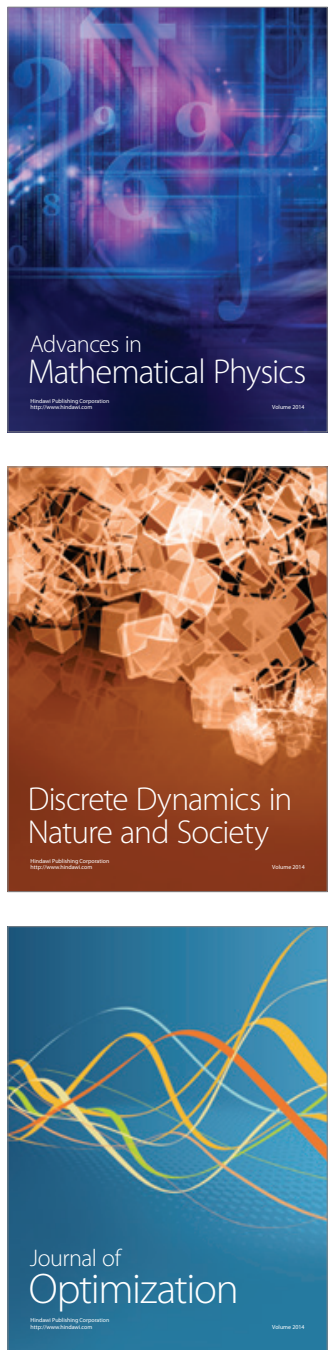